A. Manceau - D. Chateigner - W. P. Gates

\title{
Polarized EXAFS, distance-valence least-squares modeling (DVLS), and quantitative texture analysis approaches to the structural refinement of Garfield nontronite
}

\begin{abstract}
This paper reports on the new application of polarized extended X-ray absorption fine structure (PEXAFS) spectroscopy to fine-grained layer silicates taking the Garfield nontronite as a case study. Up to now application of P-EXAFS to structural studies of layer silicates has been restricted to single phyllosilicate crystals (Manceau et al. 1988; Manceau et al. 1990), but we show here that P-EXAFS can rigorously be applied to self-supporting clay films without loss of spatial resolution. The quantitative analysis of P-EXAFS requires however the preparation of highly oriented clay films, the orientation distribution of which can be assessed by texture goniometry. The Fe K-edge linear dichroism measurements were simulated by $a b$ initio EXAFS modeling performed on a nontronite cluster whose structure was refined by distance-valence least-squares calculations. It is shown that $a b$ initio modeling quantitatively accounts for the angular dependence of experimental EXAFS spectra. These calculations allowed for the identification of the fundamental character of single- and multiple-scattering paths of the photoelectron, and the structural interpretation of all spectral features observed up to $6.5 \AA$ for the in-plane and outof-plane radial structure functions of nontronite. In practice, P-EXAFS measurements allow the determination of the flattening angle of $\mathrm{Fe}(\mathrm{O}, \mathrm{OH})_{6}$ octahedra, cations distribution in the octahedral sheet with an enhanced sensitivity, and differentiation between dioctahedral and trioctahedral structures.
\end{abstract}

A. Manceau ( $)$ W. P. Gates ${ }^{1}$

Environmental Geochemistry Group, LGIT-IRIGM,

University Joseph Fourier and CNRS, F-38041 Grenoble Cedex 9, France

e-mail: Alain.Manceau@obs.ujf-grenoble.fr

D. Chateigner

Laboratoire de Cristallographie-CNRS, Université Joseph Fourier, BP166 F-38042 Grenoble Cedex 09, France

e-mail: chateign@polycnrs-gre.fr

Present address:

${ }^{1}$ Savannah River Ecology Laboratory, The University of Georgia, Drawer E., Aiken, SC 29802, USA

\section{Introduction}

Smectites have a wide occurrence in soils, weathering formations and sediments (Stucki 1988; Güven 1991), and dominate many physical, chemical and biological characteristics of systems in which they are present (Borchardt 1995). The structure of smectites consists of an octahedral sheet attached to two tetrahedral sheets (Fig. 1a). In dioctahedral structures, only two of the three symmetrically independent octahedral positions are occupied (Fig. 1b). Most smectites contain iron within the octahedral sheet (Stucki 1988), and the presence of Fe in ferric smectites provides an opportunity to probe spectroscopically the complex structure of these 2:1 dioctahedral layer silicates.

Ferric smectites are the iron-rich members of the dioctahedral bedeillite - nontronite series, and have the general structural formula $\left(\mathrm{Si}_{8-\mathrm{x}-\mathrm{y}} \mathrm{Al}_{\mathrm{x}} \mathrm{Fe}_{\mathrm{y}}^{3+}\right)\left(\mathrm{Fe}_{4-\mathrm{z}}^{3+} \mathrm{Al}_{\mathrm{z}}\right) \mathrm{Ex}_{\mathrm{x}+\mathrm{y}}^{+} \mathrm{O}_{20}$ $(\mathrm{OH})_{4} \cdot n \mathrm{H}_{2} \mathrm{O}$, where Ex represents the interlayer cation (Güven 1991). It has been shown by X-ray and electron diffraction that ferric smectites have a $C 2 / m$ layer symmetry, which indicates that octahedral cations fill cis sites (M2) (Besson et al. 1983; Tsipursky and Drits 1984) (Fig. 1b). The nontronite from Garfield has been much studied because it, together with the very rare Pfafenreuth sample (Méring and Oberlin 1967), is the only presently known representative of the ferric end-member. Its structural characteristics have been analyzed with a variety of diffraction and spectroscopic techniques, including electron and X-ray diffraction, and Mössbauer, optical, XANES, EXAFS and NMR spectroscopy (Besson et al. 1983; Bonnin et al. 1985; Tsipursky and Drits 1984). According to the currently held view, Garfield nontronite is devoid of 4-fold coordinated $\mathrm{Fe}^{3+}$, and its structural formula, calculated based on the above spectroscopic and chemical analyses, is $\left(\mathrm{Si}_{6.93} \mathrm{Al}_{1.07}\right)\left(\mathrm{Fe}_{3.97} \mathrm{Mg}_{0.01} \mathrm{Ti}_{0.02}\right) \mathrm{O}_{20}(\mathrm{OH})_{4}$ $\mathrm{Na}_{0.97} n \mathrm{H}_{2} \mathrm{O}$. Due to the large amount of data available, Garfield nontronite is a standard ferric smectite and, consequently, a material of choice for the evaluation of new structural approaches.

The main goal of this paper is to report on the new possibility offered by the application of polarized EXAFS (P- 

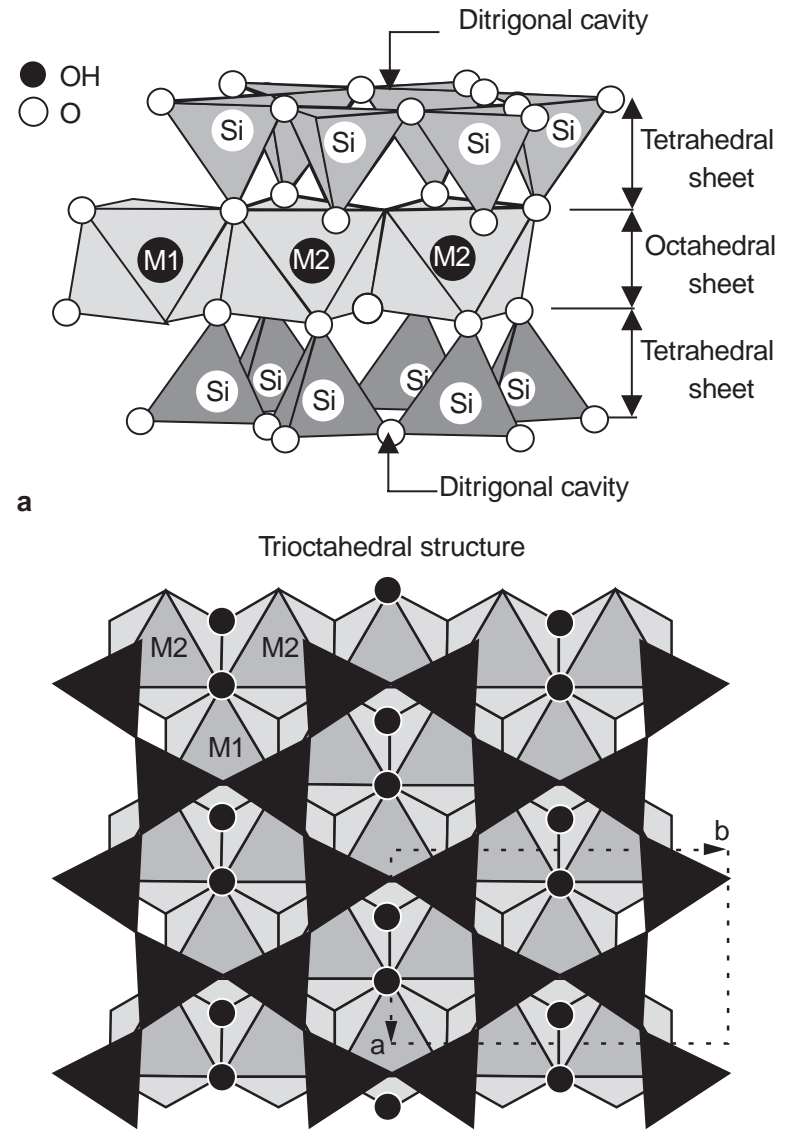

Dioctahedral structure

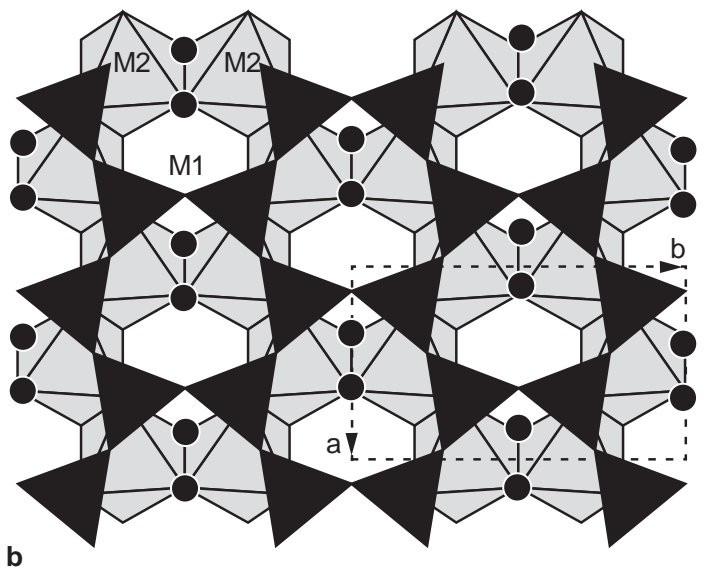

Fig. 1 a Idealized structure of nontronite. b Projection down $c^{*}$ of trioctahedral and dioctahedral layer silicates. Dark triangles are $(\mathrm{Si}, \mathrm{Al}) \mathrm{O}_{4}$ tetrahedra. One tetrahedral sheet is not shown. M1 denotes trans sites, and M2 cis sites. In dioctahedral structures the vacant M1 site is larger than M2

EXAFS) to the structural study of fine-grained layered silicates, using Garfield nontronite as a case study. Application of EXAFS to mineralogy is often limited by the strong overlap of scattering contributions from neighboring atomic shells surrounding the X-ray absorber, which restrict the spectral analysis to the 2-3 nearest shells located below $\approx 3-4 \AA$ A. Because of the highly polarized nature of the synchrotron radiation, this difficulty can be overcome for noncubic materials by analyzing the angular dependence of X-ray absorption spectra. Neighboring atoms aligned along the polarization direction are preferentially probed, while atoms located in a plane perpendicular to this direction are not observed. Using P-EXAFS to study the structure of layer silicate has the advantage of minimizing the contributions from the out-of-plane $\mathrm{Si}$ and $\mathrm{Al}$ atoms residing in the tetrahedral sheet (Fig. 1a) when the X-ray polarization vector is in the $a b$ plane of the layer. Conversely, when the polarization vector is aligned along the $c^{*}$ axis (normal to the layer plane), the contribution from octahedral layer cations vanishes.

Application of P-EXAFS to fine-grained layer silicates requires strongly textured samples, i.e., with a high degree of preferred orientation of constitutive crystallites, in order to localize accurately scattering atoms relative to the polarization direction. In the case of smectites, this can be achieved by preparing self-supporting films. In polycrystalline films, $a b$ crystallographic planes of individual platelets are preferentially aligned parallel to the film surface, but important dispersion from this orientation may occur. Obviously, the greater the in-plane alignment, the greater the resulting angular anisotropy of EXAFS spectra. Self-supporting films also differ from single crystals by the orientation of crystallites within the film. Crystallites are a priori randomly dispersed within that plane about the normal, and the film is expected to possess a $\mathrm{C}_{\infty}$ axis perpendicular to the film plane. It comes from these considerations that accurate determination of the orientation distribution (OD) of the polycrystalline film is necessary to interpret quantitatively $\mathrm{P}$ EXAFS spectra. Generally, the OD of a film can be quantified by X-ray diffraction employing a four-circle diffractometer (Schulz 1949).

In the present study, P-EXAFS spectra were interpreted by performing full multiple-scattering calculations. The relative importance of single-scattering (SS) and multiple-scattering (MS) EXAFS contributions in the in-plane and out-of-plane directions were discerned by $a b$ initio curved wave calculations by using the FEFF code (Rehr et al. 1991). In particular, we have utilized the ability of the latest version (FEFF7.02) to calculate EXAFS spectra with polarized X-rays. Ab initio computations were determined from a structural nontronite model first calculated by a combined distance-least-squares (DLS) and valencerule (VR) approach (Kroll et al. 1992). It will be shown that combination of these two theoretical approaches, $a b$ initio P-EXAFS calculations and DLS-VR simulations, in association with experimental P-EXAFS measurements is complementary, and allows for interpretation beyond the $3.3 \AA$ limit generally accepted for meaningful interpretation of EXAFS spectra of phyllosilicates. We believe this combination of techniques opens up new possibilities for medium-range structural analysis of these minerals. 


\section{Theory}

\section{Polarized EXAFS}

The amplitude of EXAFS spectra, and thus of peaks in radial structure functions (RSFs), depends on the angle made between the electric-field vector $(\boldsymbol{\varepsilon})$ of the incident radiation and the vector describing the join between absorbing and backscattering atom. In the plane-wave approximation, and for single-scattering processes, the angular dependence of EXAFS spectra can be written (Stern and Heald 1983):

$\chi(k, \theta)=\sum_{j} 3<\cos ^{2} \theta_{j}>\chi_{\text {iso }}^{j}(k)=\sum_{j} \sum_{i=1}^{N_{j}} 3 \cos ^{2}\left(\theta_{i}^{j}\right) \chi_{\text {iso }}^{j}(k)$

where $j$ denotes the number of the neighboring atomic shell, $i$ runs over all the $N_{j}$ atoms of the $j^{\text {th }}$ shell, $\theta_{i}^{j}$ is the angle between $\boldsymbol{\varepsilon}$ and the vector $\boldsymbol{R}_{i j}$ that connects the absorbing atom to the $i^{\text {th }}$ atom of the $j^{\text {th }}$ shell, and $\chi_{\text {iso }}$ is the isotropic (i.e. for a non-oriented powder) contribution of the $j^{\text {th }}$ shell. For a perfectly random powder, there is no angular variation, and $\chi(k, \theta)$ becomes: $\chi(k)=\chi(k, \theta)=\sum_{j} \chi_{\text {iso }}^{j}(k)$. In the curved-wave description of the photoelectron scattering, Eq. 1 possesses a second order term in $\sin ^{2} \theta$ (Benfatto et al. 1989), which is neglibible in the EXAFS regime of the absorption process.

It follows from Eq. 1 that P-EXAFS measurements provide directional, or orientational structure information, and can be used to probe the structure of layer silicates between two different directional limits, parallel and perpendicular to the (001) plane, by varying the angle between the self-supporting film and $\boldsymbol{\varepsilon}$. Because of the complete random distribution of individual crystallites around the normal to the film plane, $\theta_{i}^{j}$ varies from one crystallite to another. Thus, this formula cannot easily be handled, and requires transformation through the introduction of angles independent of the orientation of layers in the film plane. However, if one assumes that crystallites are perfectly axisymmetrically oriented around the film plane's normal (this will be demonstrated by texture analysis), then $\cos ^{2} \theta$ is independent of the projection of $\varepsilon$ onto the film plane, and can be averaged around the film normal (Bonnin et al. 1986). Figure 2a shows the coordinate system defining the atomic pair geometry relative to the film plane $(X, Y)$ and $\varepsilon$, for a given rotation $\alpha$ of the film around $X$. The atomic pair position is determined by the polar angle $\phi$, relative to the film normal $Z$, and the azimuthal angle $\Omega$, relative to the $X$ axis. The X-rays are incident on the $(Y, Z)$ plane and $\varepsilon$ makes an angle $\alpha$ with $Y$. Thus, $\cos \theta$ can be readily expressed as a function of the orientation of $\varepsilon$ relative to the film plane, $\alpha$, and the orientation of the atomic pair vector relative to the film normal, $\phi$ :

$\cos \theta=\left(\varepsilon \cdot \mathbf{R}_{\mathrm{ij}}\right) /\left(\varepsilon \times R_{i j}\right)$
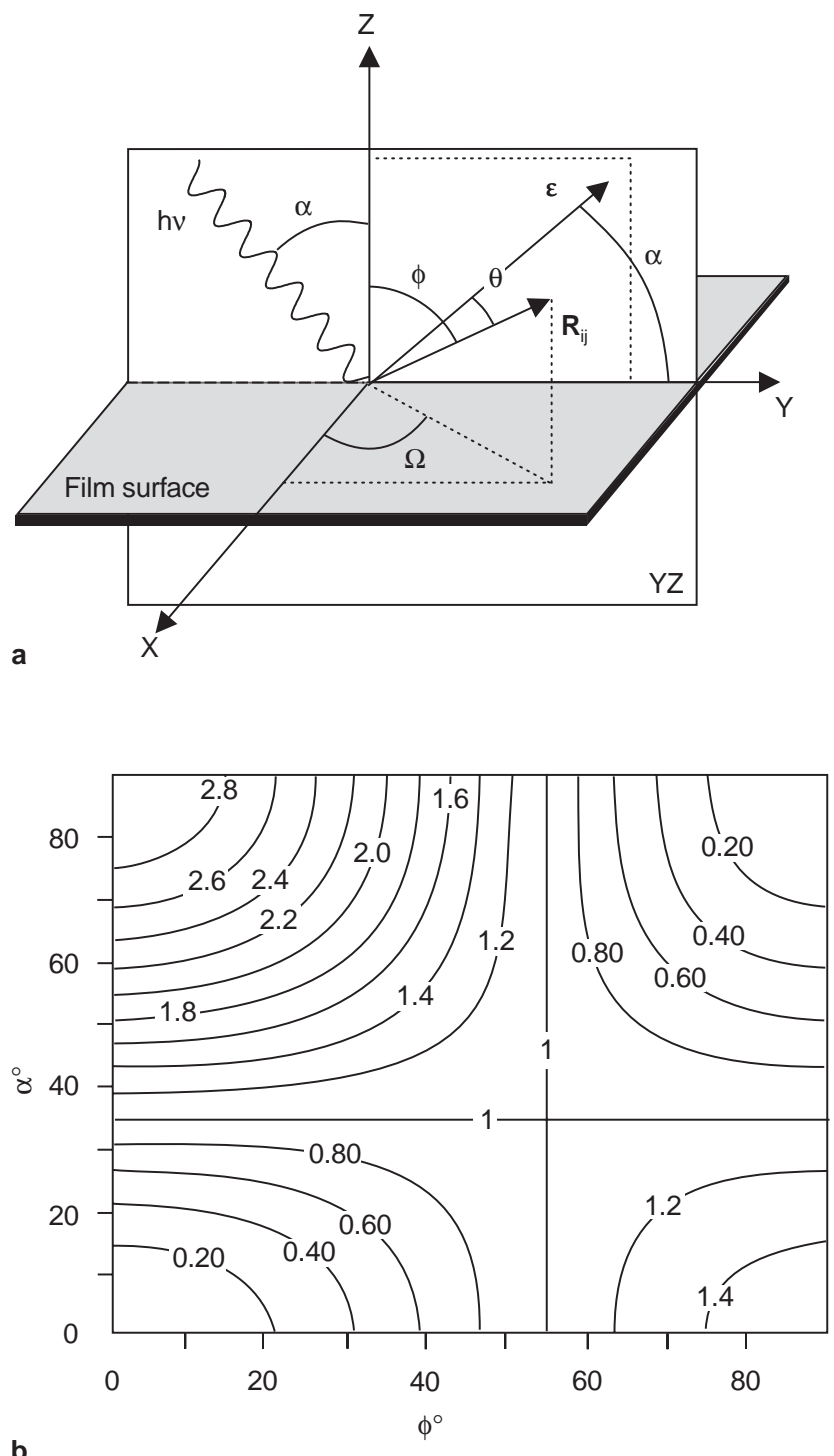

b

Fig. 2 a Coordinate system applicable to angular measurements on self-supporting films. $(X, Y)$ is the film plane, and $X$-rays are incident on the $(Y, Z)$ plane. $\alpha$ is the incidence angle between $\varepsilon$ and $X, \phi$ is the polar angle and $\Omega$ the azimuthal angle of the atomic pair $i j . \theta$ is the angle between $\varepsilon$ and the atomic pair. b Plot of the effective number of atomic neighbours $\left(N_{\text {eff }}\right)$ as a function of $\alpha$ and $\phi$ angles

$\cos \theta=\sin \phi \cos \alpha \sin \Omega+\cos \phi \sin \alpha$

$<\cos ^{2} \theta>=\frac{1}{2 \pi} \int_{0}^{2 \pi} \cos ^{2} \theta d \Omega$

replacing $\cos ^{2} \theta$ by its expression in (3) and integrating, the angle of $\boldsymbol{R}_{i j}$ describing the atomic pair relative to $\boldsymbol{\varepsilon}$ becomes:

$<\cos ^{2} \theta>=\cos ^{2} \phi \sin ^{2} \alpha+\left(\sin ^{2} \phi \cos ^{2} \alpha\right) / 2$

and the relation between the crystallographic $\left(N_{\text {cryst }}\right)$ and the effective $\left(N_{\text {eff }}\right)$ number of neighbours can be written (Manceau et al. 1990):

$N_{\text {eff }}=3 N_{\text {cryst }}\left[\cos ^{2} \phi \sin ^{2} \alpha+\left(\sin ^{2} \phi \cos ^{2} \alpha\right) / 2\right]$ 
This two-variable $N_{\text {eff }} / N_{\text {cryst }}$ function is plotted in Fig. 2 b. Observe that from knowledge of $\phi$ it is possible to calculate $N_{\text {eff }}$ for every experimental $\alpha$ angle. For $\alpha=35.3^{\circ} N_{\text {eff }}$ is independent of $\phi$ and, conversely, for $\phi=54.7^{\circ}$ it is independent of $\alpha$ because for both angles $\left\langle\cos ^{2} \theta\right\rangle=1 / 3$ and $\chi(k)=\chi(k, \theta)=\sum_{j} \chi_{\text {iso }}^{j}(k)$. Consequently, the powder EXAFS spectrum of an axisymmetrically textured solid may readily be recorded from a self-supported film oriented at $\alpha=35.3^{\circ}$ (Manceau et al. 1990). Likewise, if an atomic pair makes an angle $\phi=54.7^{\circ}$ from the sample normal, then $N_{\text {eff }}=N_{\text {crys }}: \phi=54.7^{\circ}$ is a "magic angle" and the PEXAFS amplitude of the considered pair is independent of the orientation $\alpha$ of the sample relative to the X-ray beam. For $\phi<54.7^{\circ}, \chi(k, \alpha)$ increases with $\alpha$, whereas for $\phi>54.7^{\circ}, \chi(k, \alpha)$ decreases with increasing $\alpha$.

\section{Quantitative texture analysis}

The quantitative determination of the texture is based on the concept of orientation distribution (OD) (Bunge 1981), which represents the distribution of all possible orientations of constitutive crystallites in a polycrystalline aggregate. To assess the OD function, one generally conducts X-ray, neutron or electron diffraction experiments (Wenk 1985). Such experiments consist of measuring the so-called $\{h k l\}$ pole figures, relative to the specific $\{h k l\}$ equivalent crystallographic planes of the material. With X-rays, this is in practice performed on a diffractometer, as schematically shown in Fig. 3. Figure 3a represents the three rotation movements $\theta_{h k l}, \varphi$ and $\rho$, respectively Bragg angle, azimuthal and colatitude rotations. Note that, after having fixed $\theta_{h k l}$ for the sample (and $2 \theta_{h k l}$ for the detector) suitable to obtain diffraction on the $\{h k l\}$ planes, the two other rotations conserve $\theta_{h k l}$. It is then possible to rotate the sample in every position around $\varphi$ and $\rho$ to bring successively all crystallites into a diffracting position. At each position the diffracted intensity, $I_{h k l}(\rho \varphi)$, is proportional to the number of $\{h k l\}$ planes which are in the $(-\rho,-\varphi)$ orientation in the sample. Figure $3 \mathrm{~b}$ illustrates how crystallite planes oriented $(-\rho, 0)$ within the film are brought to diffraction position by a $\rho$ rotation. In this figure, the darker $\{h k l\}$ planes diffract if a suitable X-ray beam is provided.

Once diffracted intensities have been measured for all possible $(\rho, \varphi)$ positions, they are projected onto the sample plane using an equal-area projection for qualitative visual representation and subsequent quantitative analysis of the textural order of the sample. Let us first imagine the extreme case of a sample composed of a single crystal aligned with its main crystallographic axes [100], [010] and [001] aligned parallel to the macroscopic $X Y Z$ reference frame (Fig. 4a, left). The corresponding theoretical $\{001 / 100 / 010\}$ pole figures consist of point-like high intensity regions corresponding to the $[001]^{*},[100]^{*}$ and [010]* directions (Fig. 4a, right). As an example, we show the experimental multipole figure for the (131/131), (004) and $(\overline{2} 02)$ reflections for a single crystal slab of biotite in Fig. 4b. The slab normal corresponds to the [001]* direc-

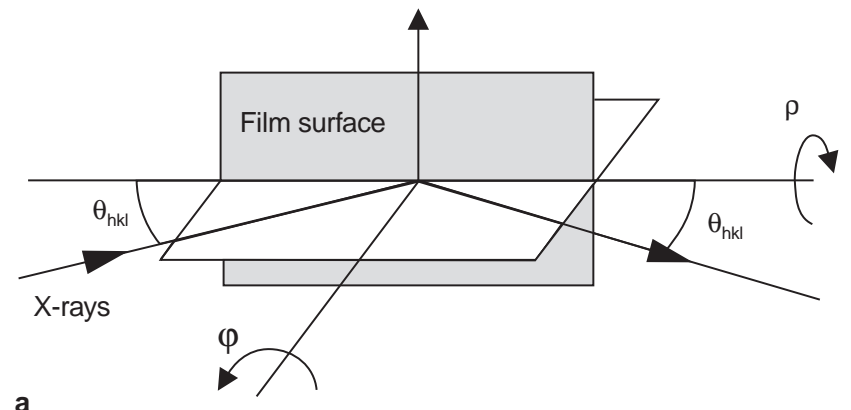

a

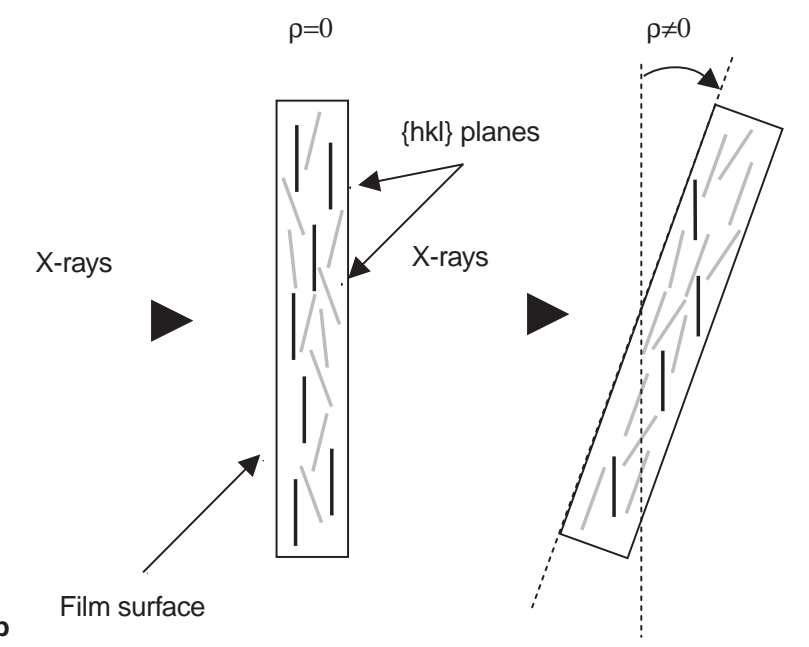

Fig. 3a, b X-ray diffraction reflection geometry used for texture analysis. a A $\{h k l\}$ pole figure is measured for a fixed $\theta_{h k l}$, while the sample is rotated by $\rho$ and $\varphi$ (both conserving $\theta_{h k l}$ ). b Side view of a showing how crystallographic planes are brought to a diffraction position by a rotation of $\rho$

tion of biotite, localised in the center of the pole figure (parallel to the $Z$ axis), which indicates that the $a b$ plane of biotite is parallel to the $X Y$ slab plane. The [131/ 131]* and $[\overline{2} 02]^{*}$ directions are localised at $\rho \approx 65^{\circ}$ from $Z$ and at $\varphi \approx 120^{\circ}$ from one another, their respective positions within the single crystal. Such pole figures could have been obtained on a polycrystalline sample with all crystallites perfectly aligned as in the case of biotite. Figure $4 \mathrm{c}$ shows how macroscopic sample and microscopic crystal reference frames are correlated. For this purpose we calculated (100), (010) and (001) theoretical pole figures of an hypothetical polycrystal having a Gaussian distribution of crystallites of $10^{\circ}$ of half width, with orthorhombic axes preferentially aligned on $X, Y$ and $Z$. The opposite extreme of Fig. 4a could be obtained on a sample without any preferred orientation (randomly distributed or powder sample) where a constant diffracted intensity is measured at any $(\rho, \varphi)$ position of the sample, yielding a homogeneous pole figure. The orientation distribution concerning our samples is between these extremes, corresponding to axially symmetric preferred orientations, for which the [001]* direction is aligned parallel to $Z$.

Normalization of diffracted intensities into pole densities $P_{h k l}(\rho, \varphi)$ is necessary to compare samples regardless 

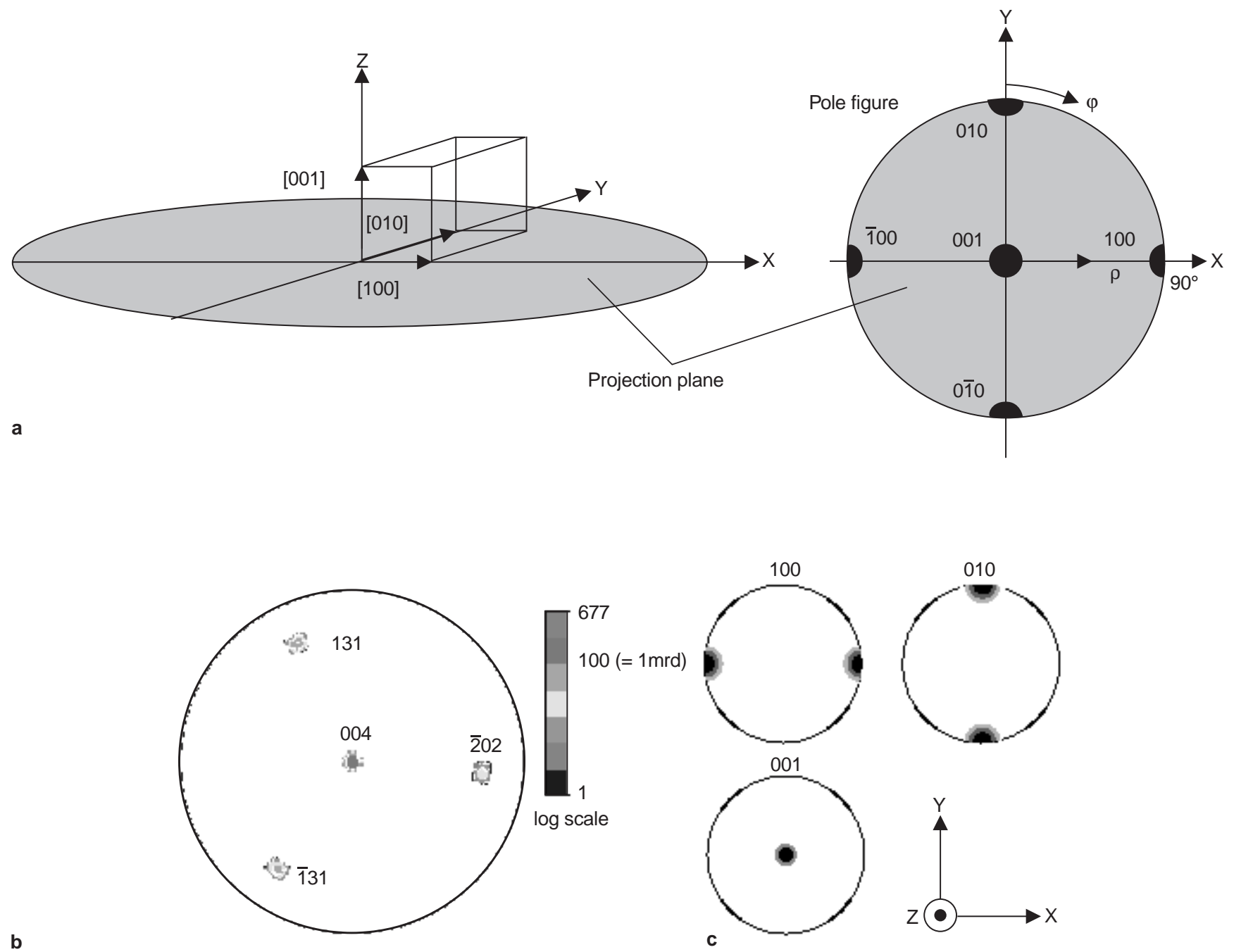

Fig. 4 a Definition of the projection plane for a pole figure, with respect to sample coordinates (left) and schematic $\{001\}$ pole figure in the case of a single crystal with cubic symmetry with main axes oriented parallel to the sample frame (right). b $\{004 / \overline{1} 31 / 131 / \overline{2} 02\}$ multipole figure of a single crystal slab of monoclinic biotite showing very narrow poles. c Theoretical $\{100\},\{010\}$ and $\{001\}$ pole figures for a Gaussian distributed texture of $10^{\circ}$ half-width (orthorhombic symmetry) with the same orientation as a

of their porosity, presence of several phases, etc., and is achieved by integrating the intensities over $\varphi$ and $\rho$, assuming that the averaged intensity of every pole figure is constant whatever the true texture is. Pole densities are expressed in mrd, or multiple of a random distribution, and equal $1 \mathrm{mrd}$ for a random sample whatever $h \mathrm{kl}$ and $(\rho, \varphi)$, while pole figures of textured samples will exhibit values above and below 1 mrd. For a single crystal (biotite of Fig. 4b) the orientation density tends to infinity for all $\{h k l\}$, limited only by experimental resolution (mainly beam divergence) and the crystal mosaic.

In smectites only a few reflections are accessible as a result of their high $d$-spacings $\left(\theta_{h k l}\right.$ is too low to ensure constant irradiation of the sample) and systematic extinctions. This prevented us from measuring a large enough sample of pole figures to ensure that all texture components were revealed, even if information for all components were fortuitously present in all pole figures. The $h k l$ reflections were also strongly overlapping because of poor 3 dimensional periodicity, i.e., turbostratic layer stacking (Eggleton 1977). Consequently, the measurable peaks consisted of several overlapped reflections, which resulted in multipole figures of complex direct interpretation. It was then absolutely necessary to calculate the complete OD from these multipole figures to obtain a quantitative description of the texture. Once the OD has been determined, every desired single pole figure can be recalculated. We used an iterative method, called WIMV, to quantify the OD (Matthies et al. 1987), which is based on the numerical refinement of the pole figure data by minimizing the reliability factors:

$\overline{R P}=\frac{1}{I} \sum_{h k l} \sum_{j} \frac{P_{h k l}^{\mathrm{calc}}(j)-P_{h k l}^{\mathrm{obs}}(j)}{P_{h k l}^{\mathrm{obs}}(j)}$

where $P^{\text {obs }}$ and $P^{\text {cal }}$ refer to the observed and recalculated intensities $(I)$ of pole figures, and $j$ is for all $\varphi P$ values. The $R P$ factors depend on the texture strength and, consequently, make comparison of the quality of the refinement 
between samples somewhat ambiguous. This drawback can be eliminated by introducing weights $(w)$ in (7), which incorporate the regular statistical distribution of the measured (obs) and recalculated (calc) diffracted intensities $I_{h k l}(\rho \varphi)$ (Chateigner et al., to be published).

$\overline{R w}=\frac{1}{I} \sum_{h k l} \sum_{j} \frac{\left[w_{h k l}^{\mathrm{obs}} I_{h k l}^{\mathrm{obs}}(j)-w_{h k l}^{\mathrm{calc}} I_{h k l}^{\mathrm{calc}}(j)\right]^{2}}{w_{h k l}^{\mathrm{calc}^{2}} I_{h k l}^{\mathrm{calc}^{2}}(j)}$

The overall texture strength is quantified with the texture index $F$ (Bunge 1981):

$F=\frac{1}{8 \pi^{2}} \sum_{g}[f(g)]^{2} \Delta g$

where $f(g)$ is the orientation distribution function, which represents the orientation distribution of the constitutive crystallites, and $\Delta g$ is the OD cell volume.

\section{DVLS}

The distance-least-squares (DLS) structural modeling, introduced by Meier and Villiger (1969) is based on the fact that the number of crystallographically independent interatomic distances in most structures exceeds the number of atomic coordinates and unit cell parameters, so that the latter may be determined by prescribing a sufficient number of the former. Recently, DLS minimizations have been applied to model the structure of the phyllosilicates, stilpnomelane and parsettensite (Bish and Von Dreele 1989; Guggenheim and Eggleton 1994). Since DLS only adjusts distances between atoms without considering local electrical neutrality, the DLS method tends to produce regular coordination polyhedra, and irregular distances must be prescribed to prevent this tendency. This limitation can be overcome by incorporating bond-valence (VR) rules into the DLS method.

The VR approach was developed by Brown and co-authors (Brown 1981, 1992; Brown and Shannon 1973), and is based on the concept of bond valence first introduced by Pauling (1929). An important VR rule states that the sum of individual bond valences formed by an ion equals the formal valence of this ion. The distance-valence leastsquares (DVLS) program developed by Kroll et al. (1992) minimizes the sum of three functions $F 1, F 2$ and $F 3$. The distance-least-squares function, $F 1$, is simply the sum over all inequivalent distances in the structure:

$$
F 1=\sum_{p q}\left[w_{p q}\left(R_{p q}^{1}-R_{p q}\right)^{2}\right]
$$

where $R_{p q}^{1}$ is the prescribed interatomic distance for the $p q$ atomic pair, $R_{p q}$ the refined distance, and $w_{p q}$ an empirical weight. Similarily, F2, the angle-least-squares function, sums all angular deviations for a given three-atom group:

$F 2=\sum_{p q r}\left[w_{p q r}\left(\varphi_{p q r}^{1}-\varphi_{p q r}\right)^{2}\right]$

Here, the angle $\varphi$ at atom $p$ is defined by the three atomic relative positions of $p, q$, and $r$, and $\varphi_{p q r}^{1}$ is the prescribed angle, $\varphi_{p q r}$ the refined angle, and $w_{p q r}$ is an empirical weight. The valence-least-squared function, $F 3$, sums over all deviations from the formal valence of a particular atom of pre-defined coordination within the given structure:

$F 3=\sum_{p}\left[w_{p}\left(V_{p}^{1}-V_{p}\right)^{2}\right]$

where $V_{p}^{1}$ is the formal valence of atom $p$, and $V_{p}$ the calculated valence. The total valence $V_{p}$ of an atom is obtained by summing its individual bond valences: $V_{p}=\sum_{q} v_{p q} \cdot v_{p q}$ values were calculated by the classical empirical expression relating $v_{p q}$ to bond lengths $R_{p q}$ (Brown and Altermatt 1985):

$v_{p q}=\exp \left[\left(R_{p q}^{0}-R_{p q}\right) / 0.37\right]$

where $R_{p q}^{0}$ is a bond length normalized to bond valence 1 . The $R_{p q}^{0}$ were determined from a systematic analysis of several thousand inorganic structures (Brese and O'Keefe 1991; Brown and Altermatt 1985; Brown and Shannon 1973).

\section{Experimental}

\section{Sample preparation}

The presence of intimately mixed Fe oxide impurities in nontronites has been noted (Güven 1991; Murad 1987), and their removal was accomplished by carefully performing successive sedimentations in deionized water. The Na-saturated $<2 \mu \mathrm{m}$ size fraction of the purified Garfield nontronite was then used. Thus, the chemical formula for Garfield in this study is considered to be close to that given by Bonnin et al. (1985). Additionally, biotite B-13 served as a trioctahedral Fe(II) reference and has been extensively studied (Manceau et al. 1988; Manceau et al. 1990).

Highly oriented self-supporting films were prepared by vacuum $(-0.8 \mathrm{MPa})$ filtration of dispersed nontronite suspensions onto $47 \mathrm{~mm}$ diameter filters (Millipore) with $0.05 \mu \mathrm{m}$ pore size. The mass of clay necessary to yield films of suitable thickness for EXAFS measurements was based on the diameter of the filter support and the total $\mathrm{Fe}$ content of the clay. After filtration, the filter with clay film was cut in half, and a $1 \mathrm{~cm}$ wide strip was cut from each half, yielding two $\approx 1 \times 4 \mathrm{~cm}$ films for $\mathrm{X}$-ray measurements (pole figures and EXAFS).

\section{Texture analysis}

The X-ray diffraction texture analysis experiments were performed using a Huber texture goniometer in the reflection mode mounted on a classical X-ray generator. We used a point-focused incident beam of $1 \mathrm{~mm}$ diameter and monochromatized iron radiation. An oscillating sample holder diminished sample inhomogeneities at the beam scale. Three multipole figures, $\left\{004 / 11^{\frac{ \pm}{3}}\right\},\{020 / 110\}$ and $\{200 / 130\}$ were measured. The intensity of individual components was not discerned due to overlap of reflections. We consequently considered the amount of overlap as unknown and resolved overlapping reflections during the OD refinement procedure. Pole figures were measured using angle increments in tilt $(\rho)$ and azimuth $(\varphi)$ of $5^{\circ}$, and angular ranges of $0 \leq \rho \leq 85^{\circ}$ and $0 \leq \varphi \leq 360^{\circ}$ for all pole figures. Data were corrected for background and defocusing, and calibrated by measurements on a standard randomly oriented sample. In order to minimize defocusing as much as possible, relatively open $(4 \mathrm{~mm})$ detecting slits were used, which ensured a constant signal 
on the random standard up to $\rho=70^{\circ}$, without significant background. The pole figures were normalized into multiple of random distribution (mrd), and the orientation distribution (OD) calculated using the WIMV algorithm of BEARTEX (Wenk et al. in press). The OD was iteratively refined up to a velocity of convergence of $0.3 \%$, and was then used to recalculate multiple and single pole figures. The comparison between experimental and recalculated pole figures permitted visual evaluation, while RP and Rw values quantified the quality of the OD refinement.

\section{P-EXAFS}

Care was taken to ensure high quality EXAFS measurements. To avoid sample thickness effects, the absorption jump across the edge $\Delta \mu x$, where $\mu$ is the absorption coefficient and $x$ is the sample thickness, typically must be lower than 1 , and the sample must be homogeneous in thickness (Manceau and Gates 1997; Stern and Kim 1981). The mass of sample sedimented in the filter was such that $\Delta \mu x=1$ at the experimental angle of $\alpha=60^{\circ}$. The second condition is easily realized with self-supporting films. The comparison of EXAFS spectra recorded on a film and on a powder sample showed that for identical recording conditions the signal to noise ratio is much improved when working with films. It is also important that the size of the particles be smaller than one absorption length in the material (Lu and Stern 1983), $\Delta \mu d<1$, where $d$ is the particle size. Thus, $d$ should be smaller than $\mu^{-1}$. At the Fe K-edge of nontronite $d$ should be lower than $40 \mu \mathrm{m}$, a condition which is amply satisfied since the clay fraction of a material is operationally defined as $<2 \mu \mathrm{m}$.

The Fe K-edge EXAFS measurements were performed on the XAFS I spectrometer at LURE (Orsay, France) using a Si (331) channel-cut monochromator. The X-ray absorption data were recorded over the $6900-8000 \mathrm{eV}$ energy range $(E)$ corresponding to a Bragg angle variation from $46^{\circ}$ to $38.5^{\circ}$. Over this angular range more than $99.9 \%$ of the synchrotron radiation is linearly polarized in the $(Y, Z)$ plane, being precisely $100 \%$ at the monochromator Bragg angle of $45^{\circ}$ (Hazemann et al. 1992). The sample was rotated around the $X$ axis (Fig. 2) with $\alpha$ equal to $0,20,35,50$ and $60^{\circ}$. Measurements were performed in transmission mode with gas ionisation chambers filled with an air/helium mixture dosed to attenuate the beam intensity by $\approx 20 \%$ before and $\approx 50 \%$ after the samples. The counting time was $5 \mathrm{~s}$ per point.

Data analysis was carried out following standard procedures (Lengeler and Eisenberger 1980): the background contribution prior to the edge jump, $\mu x_{b}(E)$, was fitted with a linear function and subtracted from the experimental spectrum $\mu x(E)$. The post-edge absorption was fitted with a smooth spline formed by three cubic polynomials to simulate the atomic-like term $\mu x_{0}(E)$. The EXAFS signal was then determined as $\chi(k)=\left[\mu x(E)-\mu x_{b}(E)-\mu x_{0}(E)\right] / \mu x_{0}(E)$. The photoelectron wave vector $\mathrm{k}$ is defined by $k=\sqrt{\left(8 m \pi^{2} / h\right)\left(E-E_{0}\right)}$, with $m$ the effective mass of the electron, $h$ the Planck constant, and $E_{0}$ the energy origin defined to be at $\Delta \mu x /$ 2 . The intensity of $\chi\left(\alpha=90^{\circ}\right)$ was calculated via linear regression as a function of $\cos ^{2} \alpha$ and extrapolation to $90^{\circ}$ by using the formula $\chi(\alpha)=\left[\chi\left(0^{\circ}\right)-\chi\left(90^{\circ}\right)\right] \cos ^{2} \alpha+\chi\left(90^{\circ}\right)$ (Brouder 1990; Heald and Stern 1977; Manceau et al. 1988). Fourier transformation of $\chi(k)$ to real space was performed using a Kaiser function window to minimize the intensity of side lobes resulting from truncation effects (Manceau and Combes 1988).

\section{Experimental results}

\section{Quantitative texture analysis}

Experimental-normalized and OD-recalculated multipole figures are presented in Fig. 5. The OD-recalculated pat-

Fig. 5 Experimental normalized and OD-recalculated pole figures (upper left to lower right in successive order) for Garfield nontronite. Experimental pole figures are satisfactorily reproduced by the computation. The dashed circle on the $\left\{004 / 11 \frac{1}{3}\right\}$ pole figure represents the $\left\{11^{\frac{1}{3}}\right\}$ trace. Due to the turbostratic structure of smectites this reflection has a very low intensity on the powder XRD pattern of nontronite, and appears as a scattering background in the prolongation of the 020 reflection. In $\{020 / 110\}$ and $\{200 / 130\}$ pole figures, zones of iso densities form regular rings, which indicates that the film possesses a $<001>^{*}$ fiber texture. Logarithmic density scale. The intensity of $\left\{11^{\frac{1}{3}}\right\}$ in the $\left\{004 / 11^{\frac{1}{3}}\right\}$ pole figure is $15 \%$

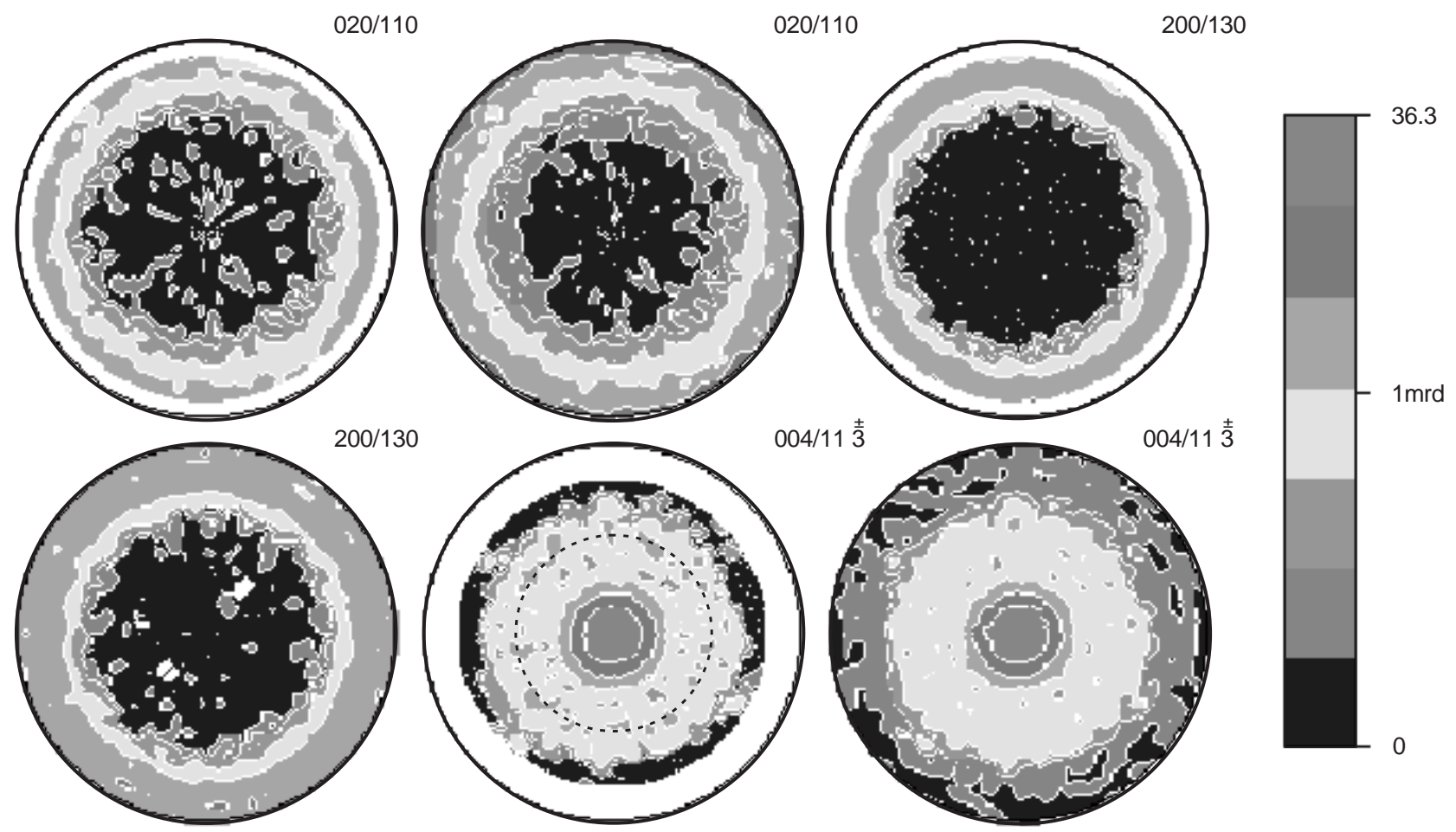


terns look very similar to the experimental patterns, which assesses the reliability of the quantitative analysis. The $\left\{11^{\frac{1}{3}}\right\}$ reflection of the $\left\{004 / 11^{\frac{ \pm}{3}}\right\}$ overlap appears as a ring at $\rho=42^{\circ}$ and is visualized by a dashed circle in Fig. 5. The presence of these spots indicates that dispersion of crystallites within the sample is low. The relative averaged deviations $R P_{1}$ and $R w_{1}$ (for density values above $1 \mathrm{mrd}$ ) are equal to $10.1 \%$ and $5.6 \%$, and $R P_{0}$ and $R w_{0}$ (for all densities) to $16.9 \%$ and $6.8 \%$. These values are fairly low, and compare favorably with recent results on strongly textured compounds having orthorhombic crystal symmetry (Chateigner et al. 1997; Wenk et al. 1996). The large difference between $R P$ and $R w$ values arises from the fact that the former factor increases with the texture strength $\mathrm{F}$, which is as high as $86 \mathrm{mrd}^{2}$ in our sample. The low value of these reliability factors allows us to place confidence in the correctness of our OD calculation. In fact, the difficulty in measuring pole figures for this clay film (disordered monoclinic compound) was only successfully overcome by using a counting time of 30 seconds per measured point.

In $\{020 / 110\}$ and $\{200 / 130\}$ pole figures, zones of iso intensities form regular rings, which is an indication of a random distribution of the crystallite $a$ and $b$ axes in the sample plane. The distribution of crystallites is thus axisymmetrical with the symmetry axis parallel to the film normal, the so called $\langle 001\rangle^{*}$ fiber texture (compare with Fig. 4b). The OD values indicated that this self-supported film contains only one orientation component.

The $\left\{004 / 11^{\frac{1}{3}}\right\}$ multipole figure does not reveal the overall maximum density of the OD since it corresponds to an overlap of reflections. Nor does it allow for a clear visualization of crystallite dispersion since individual reflections of the overlap are not localized exactly at the same $\rho$ angle. The $\{001\}$ pole figure was thus calculated from the OD (Fig. 6a). Comparison of Figs. 5 and 6a shows that the central spot is smaller in the $\{001\}$ than in the $\left\{004 / 11^{\frac{1}{3}}\right\}$ pole figure as a result of the elimination of $(11 \overline{3})$ reflections. The maximum orientation density reaches $39 \mathrm{mrd}$. To our knowledge, this is the first report of such high orientation for monoclinic self-supported films. Since the film has a fiber-like texture, the full density distribution can simply be represented by a radial section of the pole figure (Fig. 6b). The $\rho$ scan of Fig. $6 \mathrm{~b}$ has been obtained by averaging over all $\varphi$ angles the normalized intensities of the $\{001\}$ pole figure for each $\rho$ value. Examination of this figure shows that the maximum dispersion of $c^{*}$ axis is $\sim 30^{\circ}$ as beyond this value the orientation density vanishes. The full width at half maximum of the crystallite dispersion is equal to $19.8^{\circ}$, and $\rho$ at the $1 \mathrm{mrd}$ density level is $23^{\circ}$. Unfortunately, it is not possible at present to correlate quantitatively $\rho$ and $N_{\text {eff. }}$. However, the out-of-plane distribution of $c^{*}$ for individual crystallites is very low, and the difference between $N_{\text {eff }}$ estimated from the crystallographic structure assuming an idealized fabric (Eq. 6), and the real value measured by P-EXAFS should be negligible to a first approximation. For instance, $N_{\text {eff }}$ for an $\mathrm{Fe}-\mathrm{Fe}$ pair belonging to a layer disoriented by

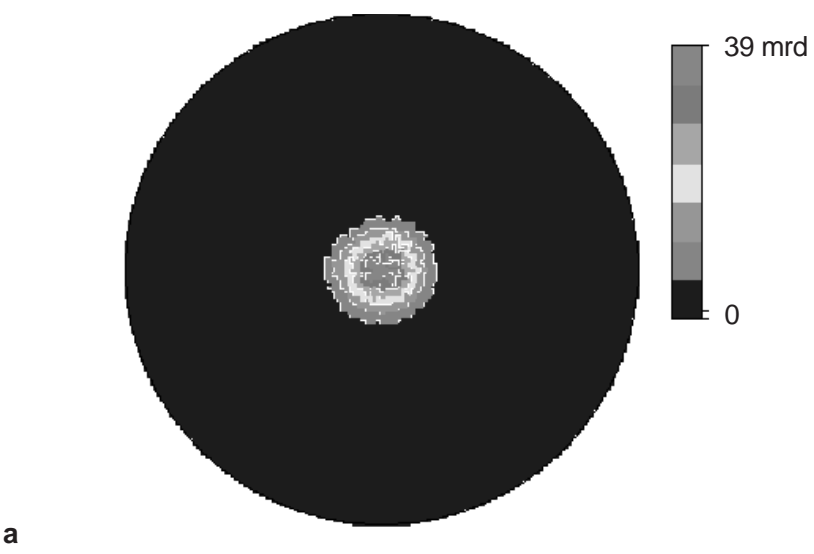

a

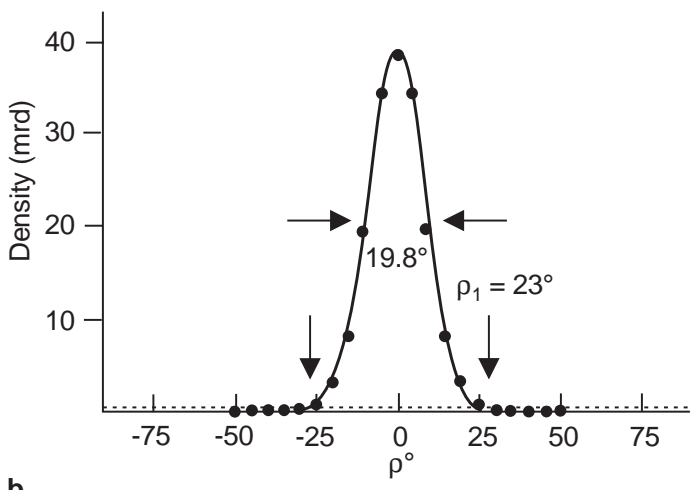

b

Fig. 6 a Nonoverlapped $\{001\}$ recalculated pole figure. b Integrated $\rho$ scan of the previous pole figure showing the angular dispersion of $c^{*}$ for the individual crystallites. The 1 mrd density level is represented by an horizontal dashed line

$19.8^{\circ} / 2=9.9^{\circ}$ is as low as 0.09 in the out-of-plane orientation as compared to zero for a perfectly oriented layer. The $N_{\text {eff }}$ for most dispersed layers $\left(\rho=30^{\circ}\right)$ is 0.75 .

\section{P-EXAFS}

$\chi\left(\alpha=90^{\circ}\right)$ calculation

Figure 7 shows the $k^{3}$-weighted P-EXAFS spectra obtained under the different polarization configurations $0^{\circ}<\alpha<60^{\circ}$. The quality of data is very high, up to nearly $14.7 \AA^{-1}$. A large dependence upon polarization is seen, consistent with a successful preparation of a highly oriented clay film. Polarization effects will be interpreted structurally in the following sections by examining Fourier transforms (FTs) of the EXAFS spectra. In the present section, the accuracy of the extrapolation method for calculating $\chi\left(\alpha=90^{\circ}\right)$ will be considered in some detail as it is the first time that this approach has been applied to the study of clays.

The $k^{3} \chi(k, \alpha)$ spectra display isosbestic points at values of $k$ for which $\chi(k)$ is independent of $\alpha$. Thus, there exist a number of $k$ values where all spectra should have precisely the same $\chi$ value. Isosbestic points are very sensitive to 


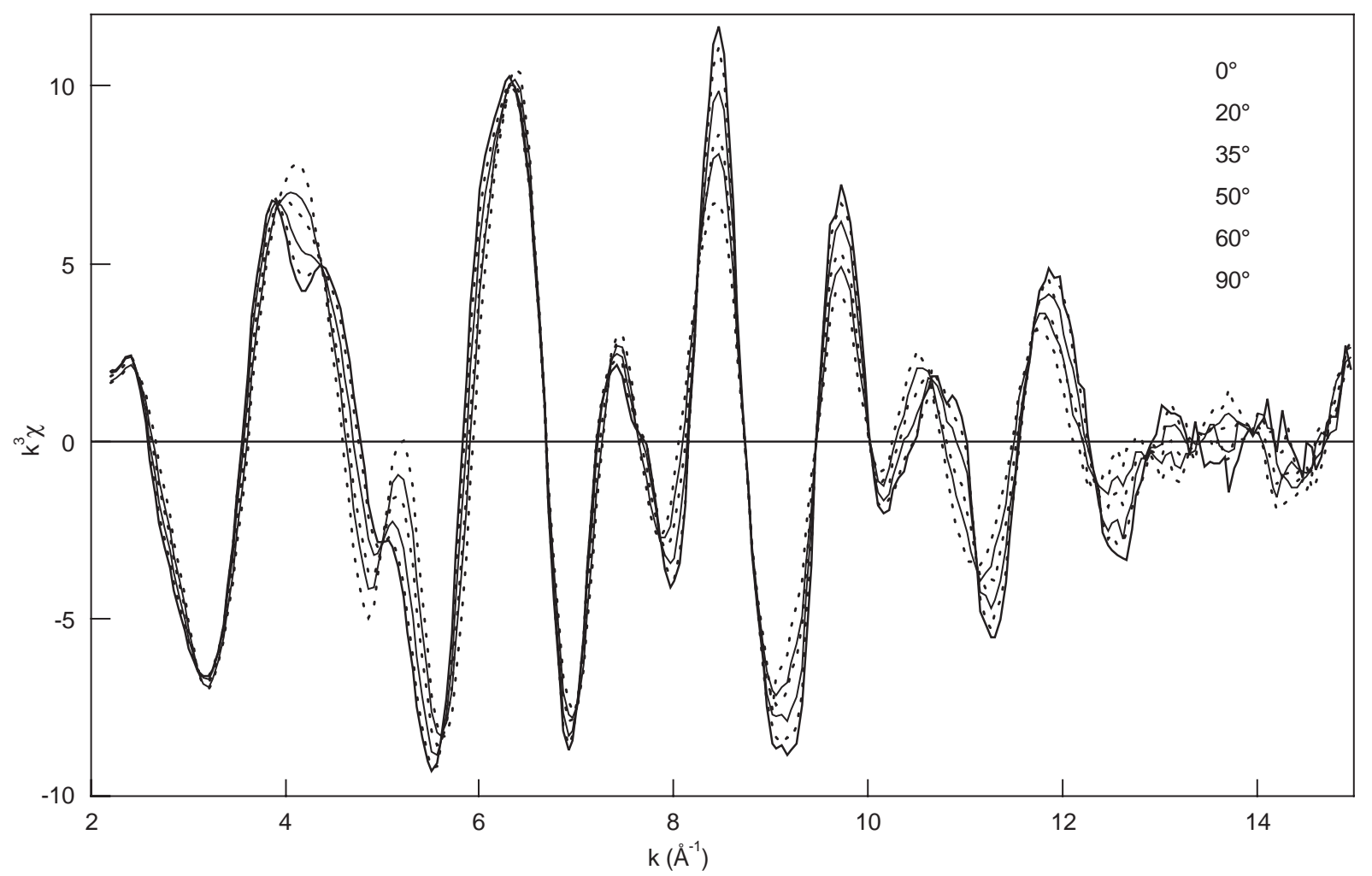

Fig. 7 The $k^{3}$-weighted Fe K-edge polarized EXAFS spectra for Garfield nontronite at $\alpha$ angles of $0,20,35,50$ and $60^{\circ}$. The $90^{\circ}$ spectrum has been obtained by regression of the experimental amplitude (see text). The amplitude of $\chi$ decreases with increasing $\alpha$ at $8.5 \AA^{-1}$, and increases with $\alpha$ at $4 \AA^{-1}$. Note the presence of isosbestic points where $\chi(k, \alpha)$ is independent of $k$

both experimental artifacts and normalization errors during the extraction of $\chi$, and thus represent a stringent test to assess the reliability of P-EXAFS measurements. Isosbestic points are observed over the entire $k$ span (Fig. 7), despite the lower signal amplitude and signal to noise ratio at the higher $k$ range.

The accuracy of P-EXAFS in detecting the anisotropy of bond vectors in clay films can be quantified by calculating $r^{2}$ linear regression coefficients for each $k$ value. The goodness of the extrapolation procedure may then be evaluated by plotting either $k^{3} \chi(\alpha)$ for specific $k$ values or $r^{2}(k)$ function (Figs. 8 and 9). Selected plots of $k^{3} \chi(\alpha)$ in Fig. 8 correspond to $k$ values where the angular variation of the signal was maximum. Note that five measurements are sufficient to calculate $\chi\left(k, \alpha=90^{\circ}\right)$ with a high accuracy: $r^{2}$ is generally greater than 0.99 , with a noticeable exception for $\chi\left(k=9.05, \alpha=35^{\circ}\right)$ where $r^{2}=0.969$. For this point the maximum deviation between measured and calculated $\chi$ is $2.5 \%$. Except in this particular situation, the error was generally below $1 \%$, and $2.5 \%$ is clearly an extreme case, which we assume to be due to experimental error in the measurement of $\mu$ at this particular energy. Examination of $r^{2}(k)$ traces represented in Fig. 9 leads to the following observations. First, $r^{2}$ values are generally greater than 0.9 for most of $k$ values.
Second, $r^{2}$ are more dispersed above $10 \AA^{-1}$ as a result of the progressive signal dumping and concomitant increased noise. Third, Fig. 9 exhibits a series of points where $r^{2}$ values are low. Explanation of this behavior is provided by comparison of $r^{2}$ with $\chi(k, \alpha)$ (Fig. 9): these sharp lines precisely coincide with the position of isosbestic points. Then, it becomes obvious that the calculation of $\chi\left(k, \alpha=90^{\circ}\right)$ has no statistical significance wherever $\chi$ is independent of $\alpha$.

Another assessment of precision for the linearization and extrapolation method is to compare experimental and recalculated spectra at particular angles. Figure 10a shows that $k^{3} \chi\left(k, \alpha=35^{\circ}\right)_{\exp }$ and $k^{3} \chi\left(k, \alpha=35^{\circ}\right)_{\text {recalc }}$ are hardly distinguishable over most of the $k$ range. As expected from the anomalous $\chi\left(k=9.05, \alpha=35^{\circ}\right)$ measurement noted previously, some spectral differences are noticeable at $k=9.05 \AA^{-1}$, but also for $k>12 \AA^{-1}$ owing to the dumping of the signal. The quality of the extrapolation may lastly be evaluated from comparison of $k^{3} \chi\left(k, \alpha=35^{\circ}\right)_{\exp }$ with the sum $2 / 3 k^{3} \chi\left(k, \alpha=0^{\circ}\right)_{\exp }+1 /$ $3 k^{3} \chi\left(k, \alpha=90^{\circ}\right)_{\text {calc }}$ as $\bar{\mu}\left(\alpha=35.3^{\circ}\right)=2 / 3 \mu_{\|}+1 / 3 \mu_{\perp}$ (Manceau et al. 1990) (Fig. 10b). This analysis demonstrates that $\chi\left(\alpha=90^{\circ}\right)$ can be calculated with a very high degree of precision from the measurement of a series of $\chi\left(\alpha<90^{\circ}\right)$ spectra.

\section{Qualitative interpretation}

Radial structure functions (RSFs) obtained by Fourier transform of the P-EXAFS spectra are shown in Fig. 11. The FTs were executed on $k$ - and $k^{3}$-weighted $\chi$ spectra. 

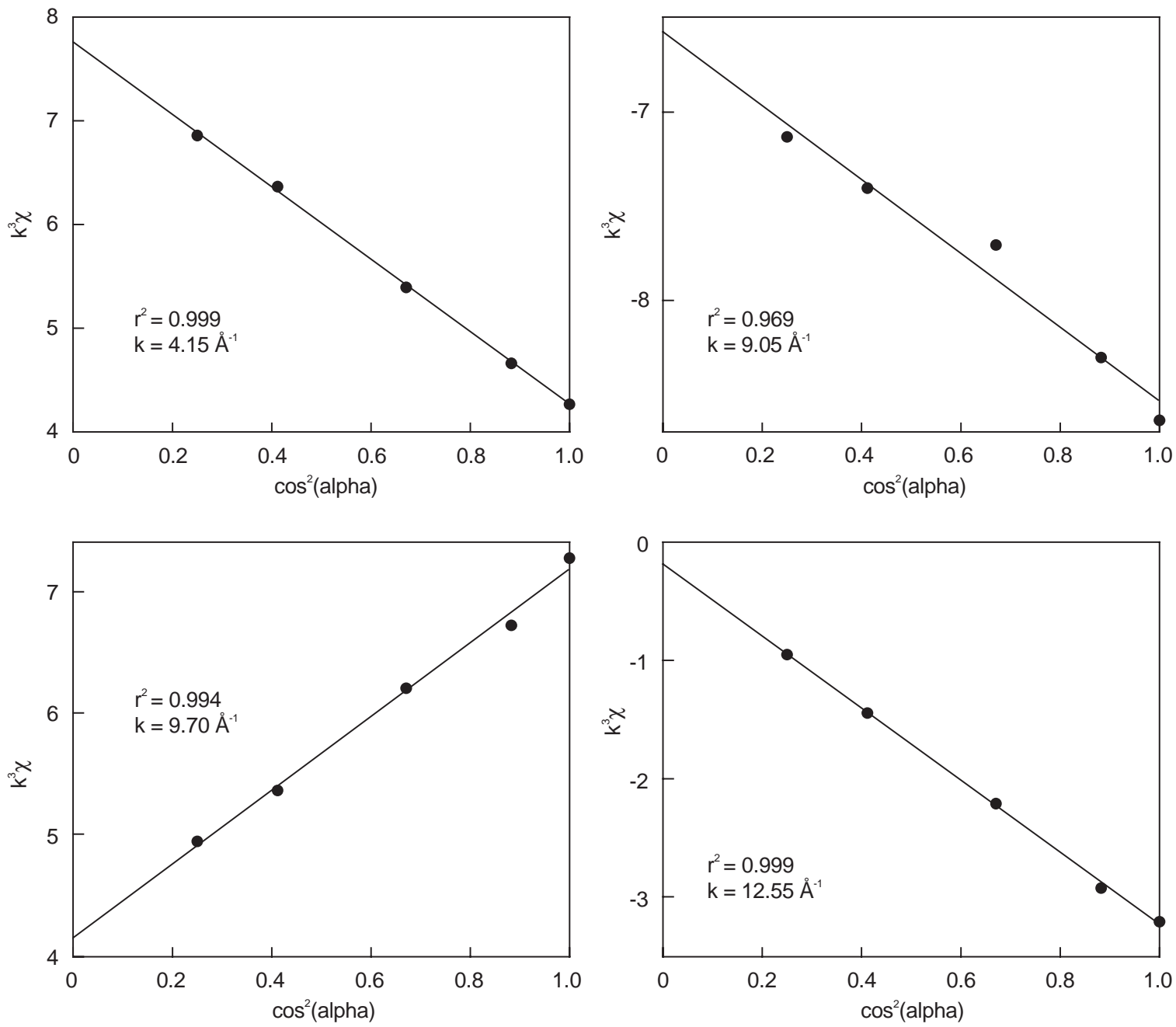

Fig. 8 Some of the regression straight lines used to calculate $\chi\left(k, \alpha=90^{\circ}\right)$ by the extrapolation procedure. Points are experimental $\chi$ values.

These different $k$-weights present the opportunity to reinforce alternatively the relative contribution of either the low $k$ or the high $k$ region in the FT. As the low $k$ region is dominated by the contribution of "light" elements (i.e., $\mathrm{O}, \mathrm{Si}$ ) and by multiple scattering (MS) paths of the photoelectron, and as the high $k$ region is dominated by the contribution of "heavy" elements (i.e., Fe), these two separate treatments may reveal weak atomic contributions that otherwise would be overlooked.

Five peaks labeled A, B, C, D and E are observed on $\mathrm{FT}(k \chi(k))$ at distances, uncorrected for phase shifts, of $R+\Delta R \approx 1.6-1.7,2.7-2.9,3.1-3.6,4.1-4.2$, and $4.8-4.9 \AA$ (Fig. 11). Peak A corresponds to the ligation $\mathrm{O}, \mathrm{OH}$ shell at the averaged crystallographic $\mathrm{R}$ distance of $2.01 \AA$, and peak $\mathrm{B}$ results from nearest in-plane $\mathrm{Fe}\left(\mathrm{Fe}_{1}\right)$ and out-of-plane $\mathrm{Si}, \mathrm{Al}\left(\mathrm{Si}_{1}\right)$ atoms at $R=3.05 \AA$ and $3.26 \AA$, respectively (Fig. 12) (Manceau et al. 1988; Manceau et al. 1990). Owing to the importance of MS paths above $R \approx 3.3 \AA$ (Chaboy and Garcia 1996; O'Day et al. 1994), more distant peaks cannot unequivocally be assigned

without theoretical computations. A good example of the need for theoretical calculations at higher values of $\mathrm{R}$ is provided by peak $\mathrm{C}$, which is resolved on $\mathrm{FT}(k \chi(k))$ but is convoluted with peak B on $\mathrm{FT}\left(k^{3} \chi(k)\right)$ plots. This difference may originate from next-nearest $\mathrm{Fe}-(\mathrm{O}, \mathrm{OH})$ pairs, or from MS paths as both types of scattering events have a maximum amplitude in the low $k$ region.

Three kinds of angular dependence are observed on Fig. 11: (1) a decrease of the intensity of RSF peaks with increasing $\alpha$ (peaks A and C); (2) the opposite trend of increasing intensity with $\alpha$ (peak D); (3) a decrease associated with a shift in distance of the peak (peaks B and E). The first situation happens when the atomic pair makes a $\phi$ angle larger than $54.7^{\circ}$, and, conversely, the second when $\phi<54.7^{\circ}$ (Eq. 6). The third situation occurs when a RSF peak contains two overlapped contributions, one with $\phi<54.7^{\circ}$ and the second with $\phi>54.7^{\circ}$. This is the case for phyllosilicates in the $\mathrm{Fe}_{1}$ shell $\left(\phi=90^{\circ}\right)$ at $3.05 \AA$ and the $\mathrm{Si}_{1}$ shell $\left(\phi=32^{\circ}\right)$ at $3.26 \AA$ : short distance $\mathrm{Fe}-\mathrm{Fe}$ pairs are maximized for the $\varepsilon_{\|}$and minimized for the $\varepsilon_{\perp}$ orientation, whereas the opposite trend is observed for the longer distance $\mathrm{Fe}-(\mathrm{Si}, \mathrm{Al})$ pairs. Manceau et al. (1990) showed that the strong anisotropy of polarization dependent $\mathrm{FT}(k \chi(k))$ functions could be used to single out the contributions coming from the octahedral $(\mathrm{Fe}-\mathrm{Fe})$ 

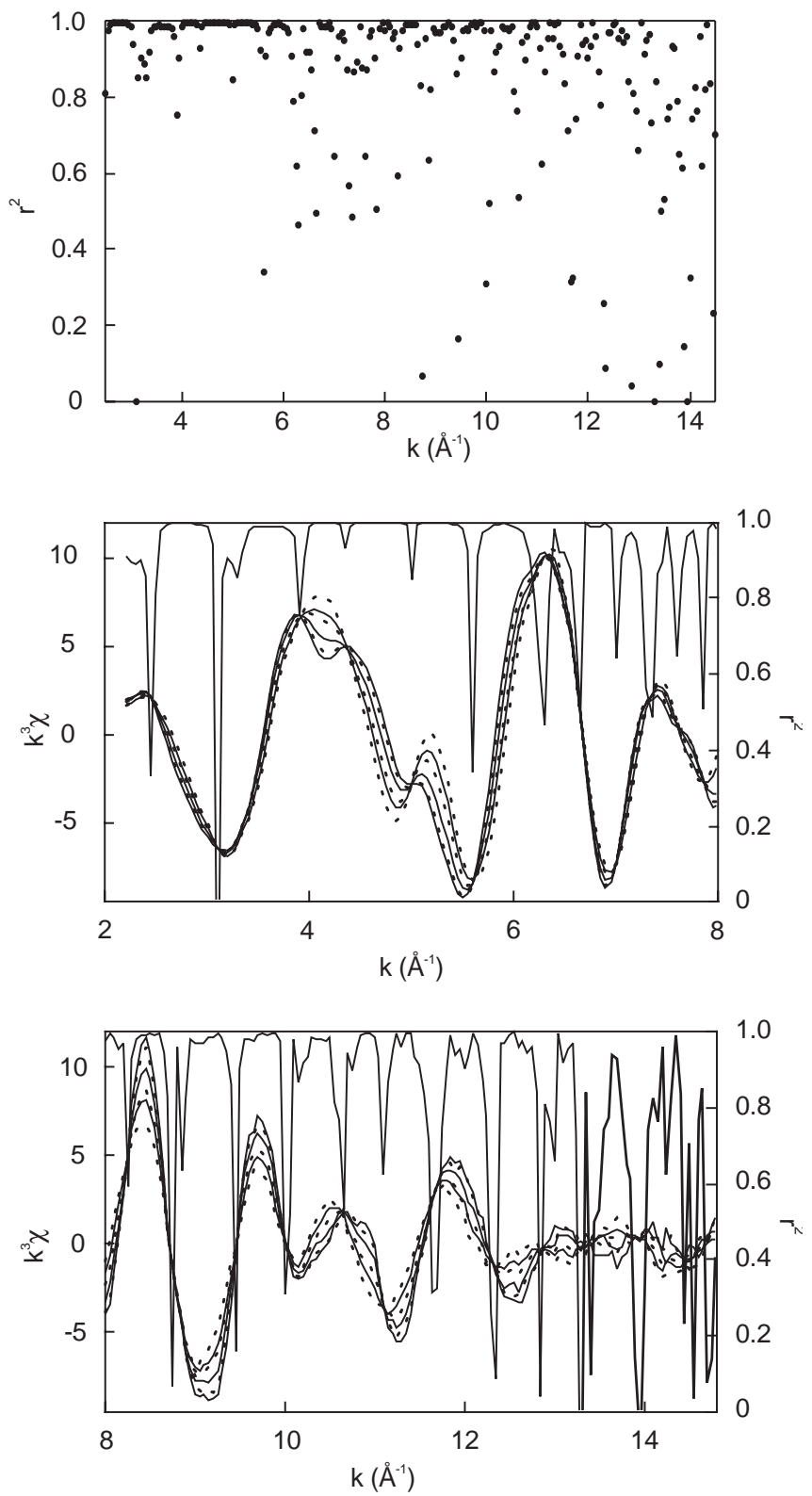

Fig. 9 The $r^{2}$ regression coefficients of determination as a function of $k$, and comparison with experimental $k^{3}$-weighted P-EXAFS spectra. The amplitude of $\chi$ decreases with increasing $\alpha$ at $8.5 \AA^{-1}$, and increases with $\alpha$ at $4 \AA^{-1}$

and from the tetrahedral [Fe- $(\mathrm{Si}, \mathrm{Al})]$ sheet of biotite crystals. Thus, the anisotropy of these two contributions to peak B allows us to probe separately, and with high sensitivity, the in-plane and out-of-plane structure of clays.

\section{First atomic shell analysis}

In edge-sharing octahedral structures, octahedra are generally flattened and the distortion is quantified by the flattening angle $\psi$ (Fig. 13a). This angle is defined as the angle between two diagonally opposite oxygens and the vertical ( $c^{*}$ direction) (Bailey 1991). Apart from very
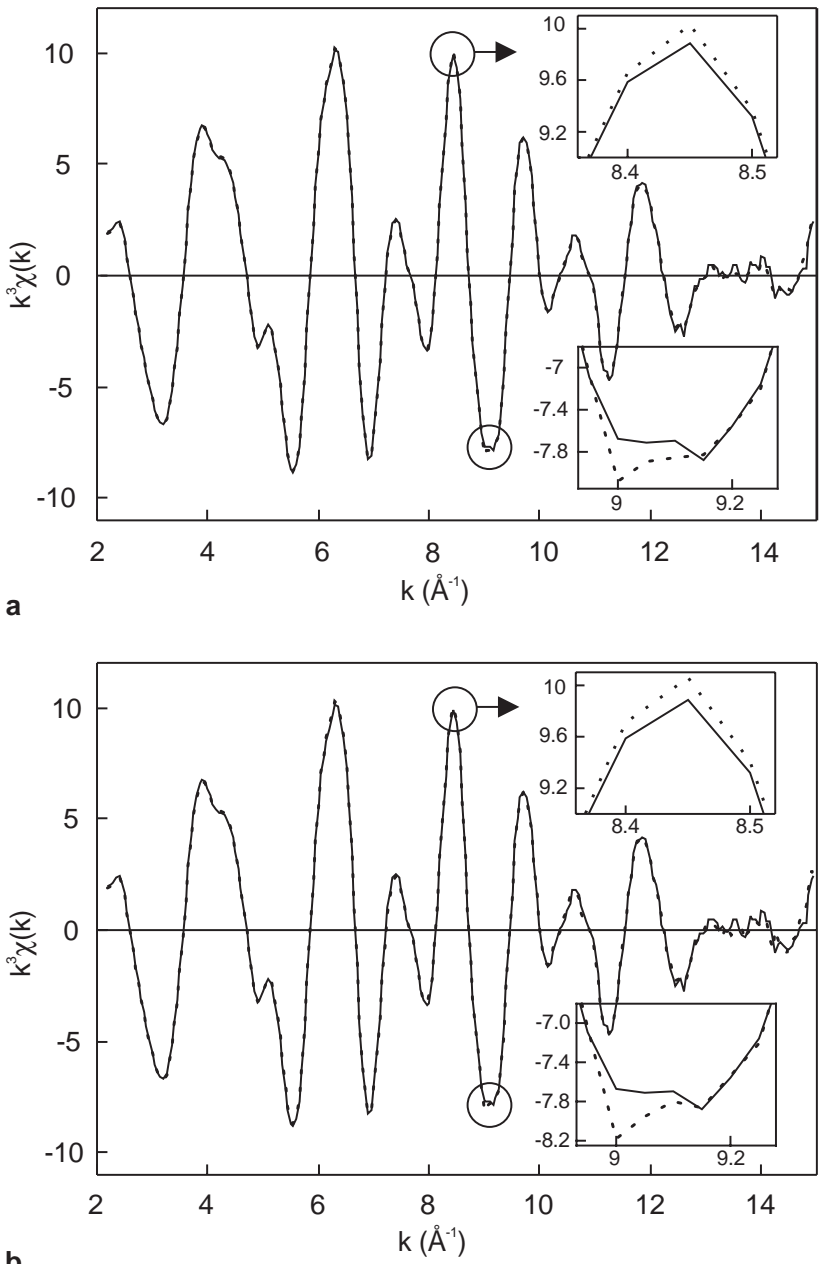

Fig. 10 a Comparison of experimental and recalculated $k^{3} \chi\left(k, \alpha=35^{\circ}\right)$. b Comparison of $k^{3} \chi\left(k, \alpha=35^{\circ}\right)_{\exp }$ with the sum 2/ $3 k^{3} \chi\left(k, \alpha=0^{\circ}\right)_{\exp }+1 / 3 k^{3} \chi\left(k, \alpha=90^{\circ}\right)_{\text {calc }}$

distorted polyhedra, this angle is identical to the average $\bar{\phi}$ angle for the six $\mathrm{Fe}-(\mathrm{O}, \mathrm{OH})$ pairs of octahedra. For instance, in the DVLS nontronite model, $\bar{\phi}=56.7^{\circ}$ and $\psi=56.6^{\circ}$. Consequently, $\psi$ can be determined from the angular dependence of the first atomic shell contribution in P-EXAFS experiments. For a perfect octahedron, $\psi=54.4^{\circ}$ (Fig. 13a) and the $\mathrm{Fe}-(\mathrm{O}, \mathrm{OH})$ contribution will be invariant with $\alpha$. Figure 11 shows that the amplitude of first RSF peaks decreases with increasing $\alpha$, which indicates that $\psi>54.7^{\circ}$ (Fig. 13a). For ideally textured selfsupporting films, this angular variation is connected to $\alpha$ and $\psi$ by (Stöhr 1992):

$\frac{I_{\alpha}}{I_{0}}=\frac{1+\frac{1}{2}\left(3 \sin ^{2} \alpha-1\right)\left(3 \cos ^{2} \psi-1\right)}{1-\frac{1}{2}\left(3 \cos ^{2} \psi-1\right)}$

where $I_{\alpha} / I_{0}$ are the peak intensities normalized to $\alpha=0^{\circ}$. The value of $\psi$ can be obtained by fitting the experimental angular dependence to theoretical curves. Results are reported in Fig. 13b together with theoretical $I_{\alpha} / I_{0}$ curves for $\psi=54.7,56.5,57,58$, and $70^{\circ}$. The second angle was 

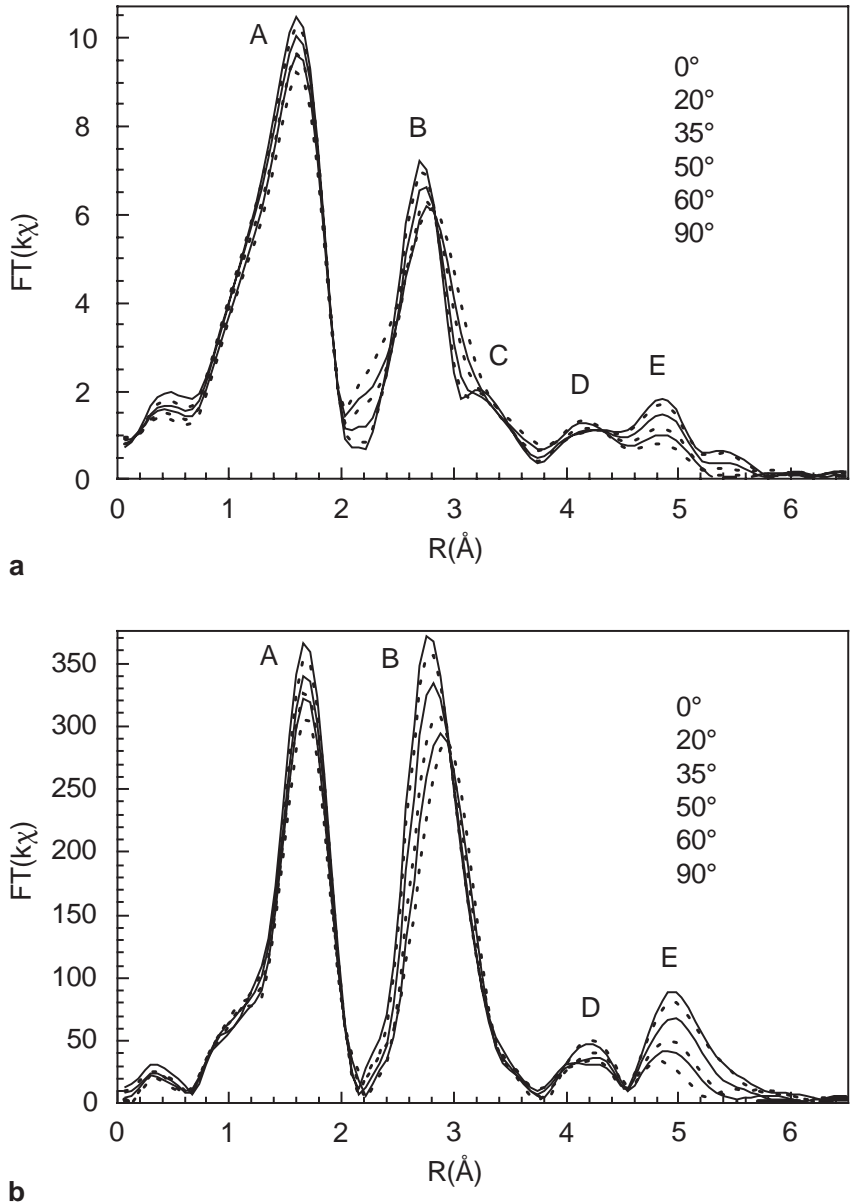

Fig. 11a, b Fe K-edge polarized RSFs for Garfield nontronite at $\alpha$ angles of $0,20,35,50,60$, and $90^{\circ}$ : a $k$-weighted Fourier transform; b $k^{3}$-weighted Fourier transform. The amplitude of peaks A, B and E decreases with increasing $\alpha$

chosen because it corresponds to the DVLS-derived value, and the others to illustrate the high sensitivity of the method. The precision on $\psi$ is about $1^{\circ}$ (Fig. 13b), provided there is good crystallite orientation. Otherwise, $\psi_{\text {exp }}<\psi_{\text {cryst }}$, and the method simply yields a minimum $\psi$ value. In the present study, given the very high orientation of the film, one can assume that $\psi_{\text {exp }}=\psi_{\text {cryst. }}$ The simulation of experimental data yielded $\psi=57-57.5^{\circ}$, which is close to $56.5^{\circ}$ determined from the DVLS model.

\section{Theoretical results}

\section{DVLS}

The Na-exchanged Garfield nontronite was assumed to have an ideal three-dimensional structure along $c^{*}$ and, consequently, DVLS modeling was done in space group $C 2 / m$ with unit cell parameters $a=5.277 \AA, b=9.14 \AA$, $c=9.78 \AA$, and $\beta=101^{\circ}$ (Besson et al. 1983; Tsipursky and Drits 1984). Parameter $c$ is equal to $10.19 \AA$ in Knontronite and was taken to $9.78 \AA$ to account for the dif- ference of size between sodium and potassium. Cell parameters were held constant while atomic coordinates were adjusted. Starting coordinates were taken from the structural model of montmorillonite (Tsipursky and Drits 1984). The atomic coordinates of $\mathrm{Fe}, \mathrm{Na}, y_{O_{2}}$ and $y_{O_{4}}$ were held invariant by special crystallographic positions. The $R_{p q}^{0}$ values were taken from Brown and Altermatt (1985). Since tetrahedra are filled by $\mathrm{Si}$ and $\mathrm{Al}$ atoms, and these nuclei are indiscernible by Fe K-edge EXAFS, $R_{(S i, A l) O}^{0}$ value was chosen as a weighted average of $R_{S i O}^{0}$ and $R_{A l O}^{0}$. The $\mathrm{Fe}-\mathrm{Si}$ interatomic distance was defined as the EXAFS value of $3.26 \AA$. No attempt was made to model the position of $\mathrm{H}$ bonded to $\mathrm{O} 4$, and the $\mathrm{O} 4-\mathrm{H}$ bond valence was fixed to 1 .

The DVLS-derived atomic coordinates, interatomic distances and bond valences are given in Tables 1 to 3 . Examination of Table 3 shows that calculated valences are very close to formal values, allowing us to place confidence in the derived interatomic distances. The consistency of this model for the layer structure of Garfield nontronite will be assessed later by comparing DVLS-derived theoretical to experimental P-EXAFS spectra.

In most phyllosilicates, the ideal lateral dimensions of the tetrahedral sheet are larger than those of the octahedral sheet causing a misfit in the structure. This misfit is mainly relieved by an in-plane rotation of adjacent tetrahedra in opposite directions around an axis normal to the layer, destroying the ideal hexagonal-sheet configuration and forming pseudoditrigonal distortion in the ring of tetrahedra (Bailey 1984). As shown in Fig. 12a, the direction of tetrahedral rotation is governed by the attraction of basal $\mathrm{O} 3$ oxygens to the nearest Fe at $3.42 \AA$. This causes the $\mathrm{O} 2$ oxygens located on the mirror plane to move in toward or out from the center of a given 6-fold cavity, in the direction of the nearest octahedral vacant site. The rotation angle $\varphi$ is a measure of the misfit, and is generally greater for dioctahedral than trioctahedral species as the former contain trivalent $\left(\mathrm{Fe}^{3+} / \mathrm{Al}^{3+}\right)$ and the latter divalent $\left(\mathrm{Mg}^{2+}\right)$ cations (Fig. 1b). The DVLS-derived $\varphi$ angle is $7.7^{\circ}$, which is a normal value for dioctahedral layer silicates (Bailey 1984; Güven 1991). Rotation around $c^{*}$ preserves the $\phi$ angle, and thus does not modify the pleochroicity of $\mathrm{O} 3_{2}$ and $\mathrm{O} 2{ }_{1}$ shells.

The difference of size between the tetrahedral and octahedral sheet is also partly compensated in dioctahedral structures by a tilt of basal oxygens out of the (001) plane creating a corrugation of the layer in surface (Lee and Guggenheim 1981) (Fig. 12c). Oxygens approximately above a vacant octahedral site $(\mathrm{O} 2)$ move down by $\Delta z=0.1$ to $0.2 \AA$ (Bailey 1984). As illustrated in Fig. $12 \mathrm{~b}$, this displacement lengthens to $3.42 \AA$ the $\mathrm{O} 1-\mathrm{O} 1$ distance between adjacent apical oxygens linked to two edge-sharing $\mathrm{Fe}$ octahedra, and reduces to $2.88 \AA$ the distance between apical oxygens linked to the same octahedral edge along [130] and [1330]. The splitting of the O1-O1 distances allows the tetrahedral sheet to adapt to the vacant site (M1) along $b$. The corrugation effect modifies the $\phi$ angle of the $\mathrm{O} 2{ }_{1}$ shell, and splits the $\mathrm{Fe}-\mathrm{O} 2_{1}$ and 


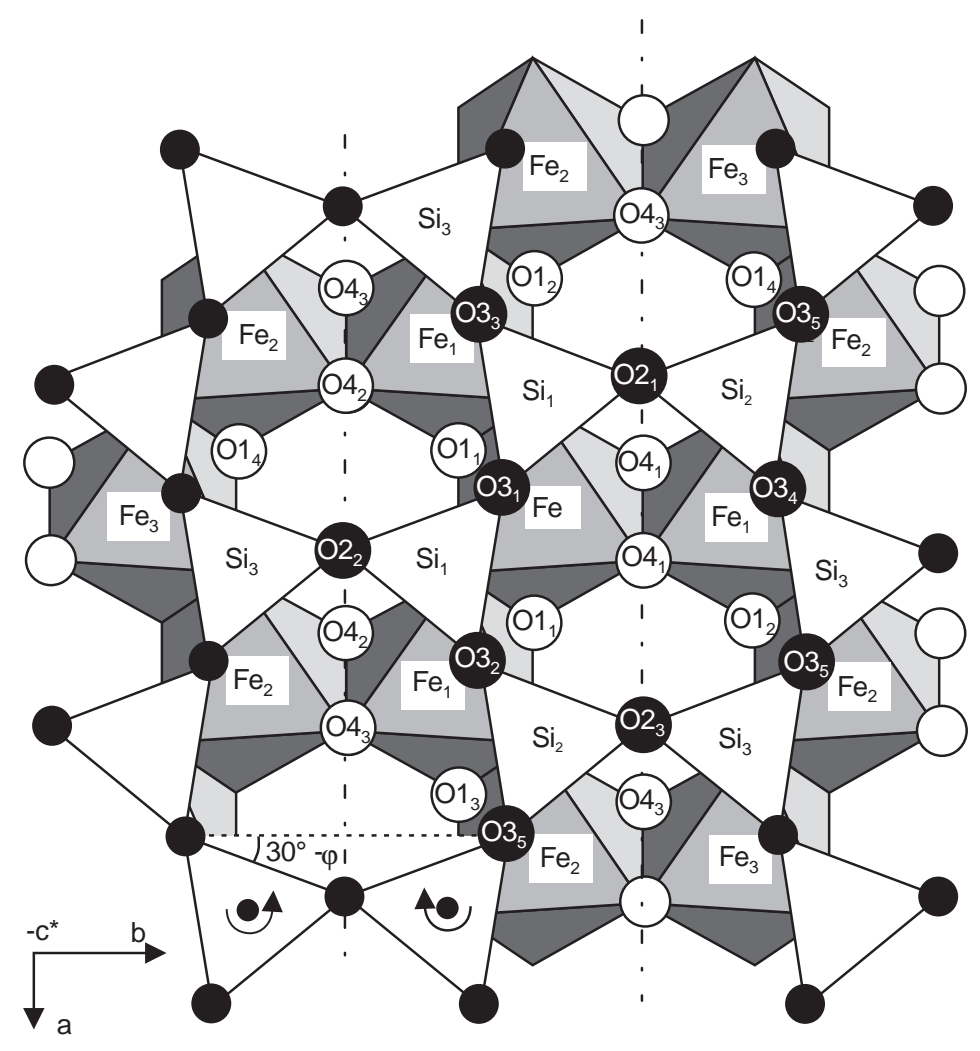

Fig. 12 a Representation down $c^{*}$ of the successive atomic shells around $\mathrm{Fe}$ in the structure of nontronite. b Splitting of O1-O1 distances in dioctahedral structures in response to the presence of a large M1 site. One tetrahedral sheet is not shown. c Representation of the biotite and nontronite structure down [100]. Dioctahedral structures have a flat and trioctahedral structures a corrugated basal plane

$\mathrm{Fe}-\mathrm{O} 3_{2}$ distances, thus influencing the pleochroism of this shell. In our DVLS structural model, the $\mathrm{O} 2{ }_{1}$ and $\mathrm{O}_{2}$ subshells were found to be at 4.03 and $4.21 \AA$ $(\Delta z=0.22 \AA)$ from the nearest $\mathrm{Fe}$ atom, which is consistent with values reported for dioctahedral structures (Bailey 1984).

\section{Ab-initio EXAFS modeling}

\section{FEFF simulations}

The FEFF 7.02 simulations were carried out on the DVLS-derived structural model for nontronite with the following flags enabled: $S_{0}^{2}$ (amplitude reduction factor) $=0.85$ O'Day et al. 1994), $R_{\max }=8 \AA$, NLEG $=5$ (up to five successive scattering paths with total distances $\leq 8 \AA$ were evaluated). Parameter $S_{0}^{2}$ is independent of the angle of X-ray incidence (Bauer et al. 1996; Lederer
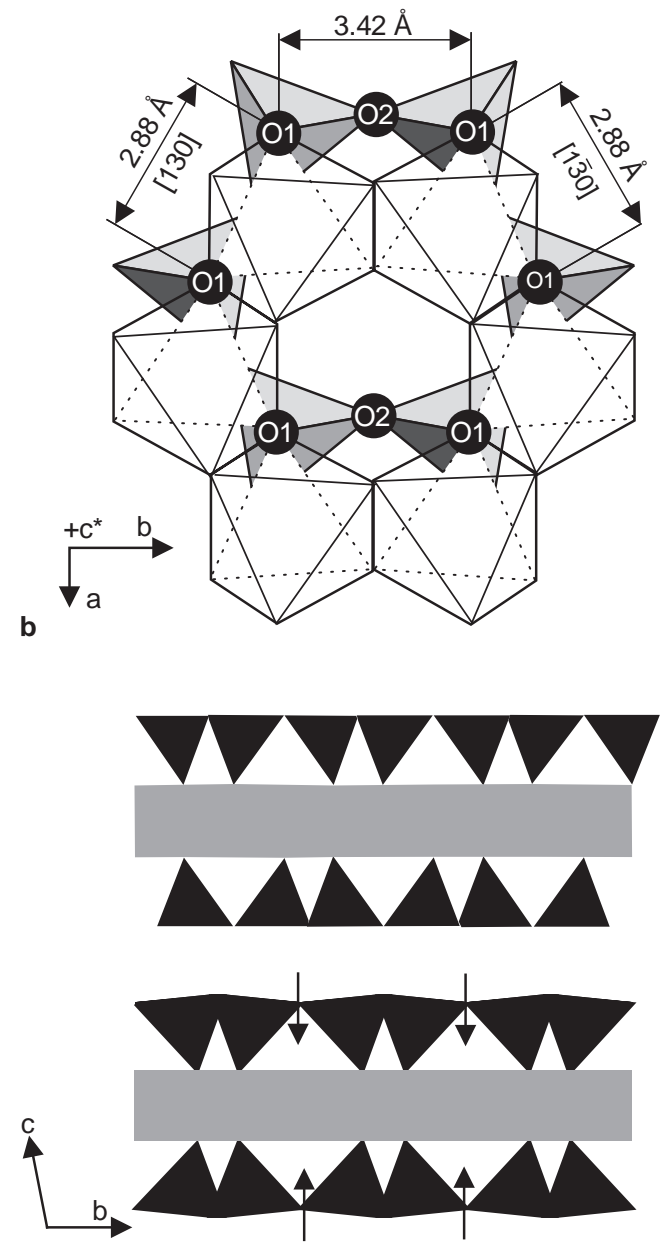

c

et al. 1993) and was kept constant for all spectra. The total number of paths is very large but only a small subset proved to be needed to reproduce EXAFS features (O’Day et al. 1994). Paths falling below a cut-off in magnitude of $6 \%$ of the mean amplitude of the first $\mathrm{Fe}-(\mathrm{O}, \mathrm{OH})$ shell were excluded with no significant effect on the quality of fit. Debye-Waller factors $(\sigma)$, accounting for atomic disorder, were set to zero for the FEFF simulations, and were subsequently adjusted to fit theory to the experimental $\alpha=35^{\circ}$. A minimum number of five $\sigma_{j}$ values proved necessary to yield a good spectral match: $\sigma_{1}^{S S}=\sigma_{2}^{S S}=0.069 \AA$ for $\mathrm{O}_{1}$ and $\mathrm{Fe}_{1}$ shells, $\sigma_{3}^{S S}=0.074$ $\AA$ for $\mathrm{Si}_{1}, \sigma_{n}^{S S}=0.10 \AA$ for single-scattering (SS) paths of more distant shells, and $\sigma_{n}^{M S}=0.11 \AA$ for all MS paths. The disorder term for SS paths was found logically to increase with distance from the central atom, and $\sigma^{M S}>\sigma^{S S}$, as expected from theory (Rehr et al. 1992). Disorder terms, $\sigma$, are intrinsic values characteristic of the solid and, consequently, do not vary with $\alpha$.

The $\mathrm{FT}\left(k^{3} \chi(k)\right)$ functions obtained by the simulations are compared in Fig. 14a, and contrasted to experimental data in Fig. 14b-e. The oscillating curve is the imaginary part of the FT, and the envelope of this curve is the amplitude, $\quad \mathrm{RSF}=\left[\operatorname{Im}(\mathrm{FT})^{2}+\operatorname{Re}(\mathrm{FT})^{2}\right]^{1 / 2}$. Examination of Fig. 14 shows that the polarization dependence of individual FT $\left(k^{3} \chi(k)\right)$ features is well reproduced by these calcu- 
a
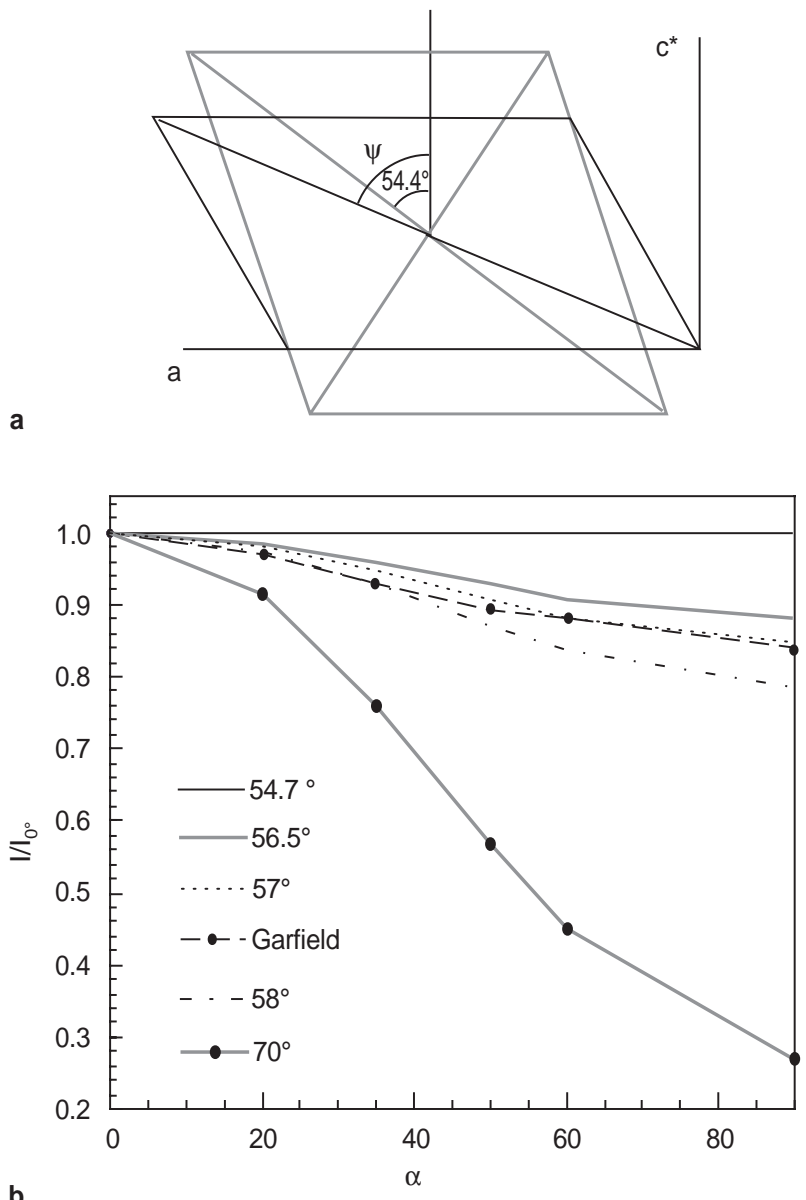

b

Fig. 13 a Projection of octahedron onto (010) before flattening (gray line), and after flattening (black line). b Normalized intensity of $\mathrm{Fe}-(\mathrm{O}, \mathrm{OH}) \mathrm{RSF}$ peaks as a function of $\alpha$, and curves calculated for flattening angles of $\psi=54.7,56.5,57,58$, and $70^{\circ}$

Table 1 DVLS-derived atomic coordinates for Na-exchanged Garfield nontronite assuming a perfect, defect-free, layer stacking. Space group: $C 2 / m . a=5.277 \AA, b=9.14 \AA, c=9.78 \AA, \beta=101^{\circ}$

\begin{tabular}{llll}
\hline Atom & $x$ & $y$ & $z$ \\
\hline $\mathrm{Fe}$ & 0.0 & 0.667 & 0.0 \\
$(\mathrm{Si}, \mathrm{Al})$ & 0.431 & 0.328 & 0.288 \\
$\mathrm{O} 1$ & 0.364 & 0.313 & 0.115 \\
$\mathrm{O} 2$ & 0.489 & 0.5 & 0.330 \\
$\mathrm{O} 3$ & 0.186 & 0.731 & 0.353 \\
$\mathrm{O} 4$ & 0.396 & 0.0 & 0.115 \\
$\mathrm{Na}$ & 0.5 & 0.0 & 0.5 \\
\hline
\end{tabular}

Table 3 Bond-valence values for Garfield nontronite; $w_{i j}=1.0$ for all pairs except $w_{\mathrm{NaO}}=0.0$

\begin{tabular}{|c|c|c|c|}
\hline & $\begin{array}{l}\text { Bond- } \\
\text {-valence } v_{p q}\end{array}$ & $\begin{array}{l}\text { Calculated } \\
\text { valence } V_{p}\end{array}$ & $\begin{array}{l}\text { Formal } \\
\text { valence }\end{array}$ \\
\hline$(\mathrm{Si}, \mathrm{Al})-\mathrm{O} 1(\mathrm{x} 1)$ & 0.90 & 3.83 & 3.87 \\
\hline$(\mathrm{Si}, \mathrm{Al})-\mathrm{O} 3(\mathrm{x} 2)$ & 0.97 & & \\
\hline$(\mathrm{Si}, \mathrm{Al})-\mathrm{O} 2(\mathrm{x} 1)$ & 0.98 & & \\
\hline $\mathrm{Fe}-\mathrm{O} 4(\mathrm{x} 2)$ & 0.48 & 3.02 & 3 \\
\hline $\mathrm{Fe}-\mathrm{O} 1$ (x2) & 0.46 & & \\
\hline $\mathrm{Fe}-\mathrm{O} 1(\mathrm{x} 2)$ & 0.57 & & \\
\hline $\mathrm{O} 1-\mathrm{Si}$ & 0.90 & 1.93 & 2 \\
\hline $\mathrm{O} 1-\mathrm{Fe}$ & 0.46 & & \\
\hline $\mathrm{O} 1-\mathrm{Fe}$ & 0.57 & & \\
\hline $\mathrm{O} 2-\mathrm{Si}(\mathrm{x} 2)$ & 0.98 & 2.03 & 2 \\
\hline $\mathrm{O} 2-\mathrm{Na}$ & 0.02 & & \\
\hline $\mathrm{O} 2-\mathrm{Na}$ & 0.05 & & \\
\hline $\mathrm{O} 3-\mathrm{Si}$ (x2) & 0.97 & 2.03 & 2 \\
\hline $\mathrm{O} 3-\mathrm{Na}$ & 0.06 & & \\
\hline $\mathrm{O} 3-\mathrm{Na}$ & 0.03 & & \\
\hline $\mathrm{O} 4-\mathrm{H}$ & 1.00 & 1.96 & 2 \\
\hline $\mathrm{O} 4-\mathrm{Fe}(\mathrm{x} 2)$ & 0.48 & & \\
\hline $\mathrm{Na}-\mathrm{O} 3(\mathrm{x} 4)$ & 0.06 & 0.5 & 1 \\
\hline $\mathrm{Na}-\mathrm{O} 3$ (x4) & 0.03 & & \\
\hline $\mathrm{Na}-\mathrm{O} 2$ (x2) & 0.05 & & \\
\hline $\mathrm{Na}-\mathrm{O} 2$ (x2) & 0.02 & & \\
\hline
\end{tabular}

lations. The position, amplitude and phase (Im part) of all contributions are quantitatively reproduced to a high degree of accuracy for all the $\alpha$ angles. Extinction of the $\mathrm{Fe}-\mathrm{Fe}_{1}$ contribution at $\alpha=90^{\circ}$ is validated by the phase and amplitude match of second $\mathrm{FT}\left(k^{3} \chi(k)\right)$ peaks in the experiment and theory (Fig. 14e). As indicated by Fig. 14a, a residual contribution of the in-plane $\mathrm{Fe}_{1}$ shell at $\alpha=90^{\circ}$ for $\mathrm{FT}\left(k^{3} \chi(k)\right)_{\exp }$ would have enhanced and shifted to shorter distances peak $\mathrm{B}$ relative to $\mathrm{FT}\left(k^{3} \chi(k)\right)_{\text {th. }}$. The extinction of this shell at $\alpha=90^{\circ}$ is an indication of the high orientation of platelets in the film plane, and is fully consistent with results from texture goniometry. This analysis shows that the quality of the film preparation in terms of texture can also be checked from P-EXAFS measurements by comparing experimental and theoretical $\operatorname{FT}\left(k^{3} \chi(k, \alpha)\right)$ functions calculated for a perfect orientation of clay layers.

It is important to emphasize that successful spectral agreement between $a b$ initio simulations and experimental data was obtained with virtually no fitting. Only a few Debye-Waller parameters were adjusted by trial and error, and these $\sigma$ values are in agreement with those previously reported for clays (Manceau et al. 1988, 1990;
Table 2 Successive atomic shells and interatomic distances from $\mathrm{Fe}$

\begin{tabular}{|c|c|c|c|c|c|}
\hline Shell & Distance (A) & Shell & Distance (A) & Shell & Distance (A) \\
\hline $\mathrm{O}_{1}(\mathrm{x} 4)$ & $1.97-2.04$ & $\mathrm{O}_{2}(\mathrm{x} 2)$ & 4.21 & $\mathrm{O}_{4}(\mathrm{x} 4)$ & $4.94-4.97$ \\
\hline $\mathrm{O} 4_{1}(\mathrm{x} 2)$ & 2.03 & $\mathrm{O} 22_{2}(\mathrm{x} 2)$ & 4.44 & $\mathrm{O}_{4}(\mathrm{x} 2)$ & 4.98 \\
\hline $\mathrm{Fe}_{1}(\mathrm{x} 3)$ & 3.05 & $(\mathrm{Si}, \mathrm{Al})_{2}(\mathrm{x} 4)$ & 4.49 & $\mathrm{Na}_{1}(\mathrm{x} 2)$ & 5.12 \\
\hline$(\mathrm{Si}, \mathrm{Al})_{1}(\mathrm{x} 4)$ & 3.26 & $\mathrm{O}_{3}(\mathrm{x} 2)$ & 4.60 & $\mathrm{Fe}_{2}(\mathrm{x} 6)$ & 5.28 \\
\hline $\mathrm{O} 3_{1}(\mathrm{x} 2)$ & 3.45 & $\mathrm{O}_{3}(\mathrm{x} 4)$ & $4.62-4.68$ & $(\mathrm{Si}, \mathrm{Al})_{3}(\mathrm{x} 8)$ & $5.38-5.45$ \\
\hline $\mathrm{O}_{4}(\mathrm{x} 2)$ & 3.74 & $\mathrm{O}_{4}(\mathrm{x} 2)$ & 4.70 & $\mathrm{O}_{5}(\mathrm{x} 6)$ & $5.72-6.02$ \\
\hline $\mathrm{O}_{2}(\mathrm{x} 4)$ & $3.74-3.82$ & $\mathrm{O} 2_{3}(\mathrm{x} 2)$ & 4.83 & $\mathrm{Na}_{2}$ (x2) & 5.93 \\
\hline $\mathrm{O} 2_{1}(\mathrm{x} 2)$ & 4.03 & $\mathrm{O} 4_{3}(\mathrm{x} 2)$ & 4.89 & $\mathrm{Fe}_{3}(\mathrm{x} 3)$ & 6.10 \\
\hline
\end{tabular}



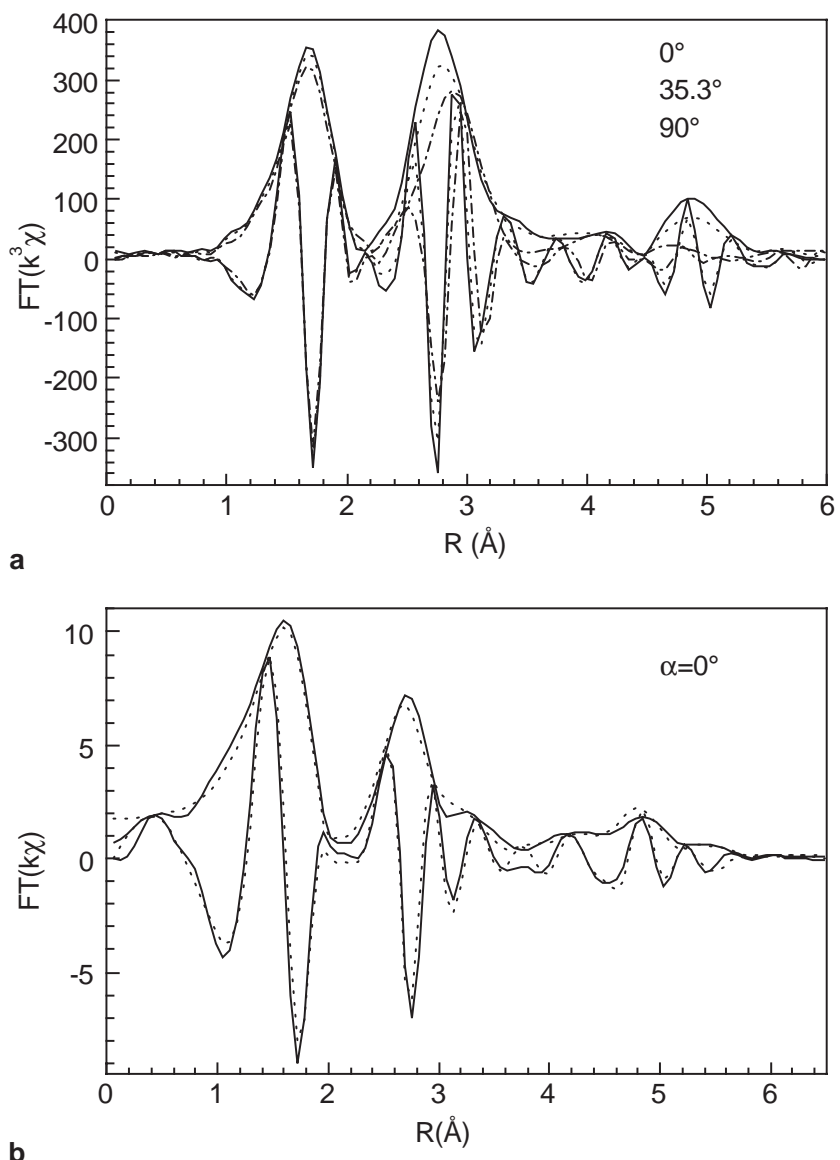

b

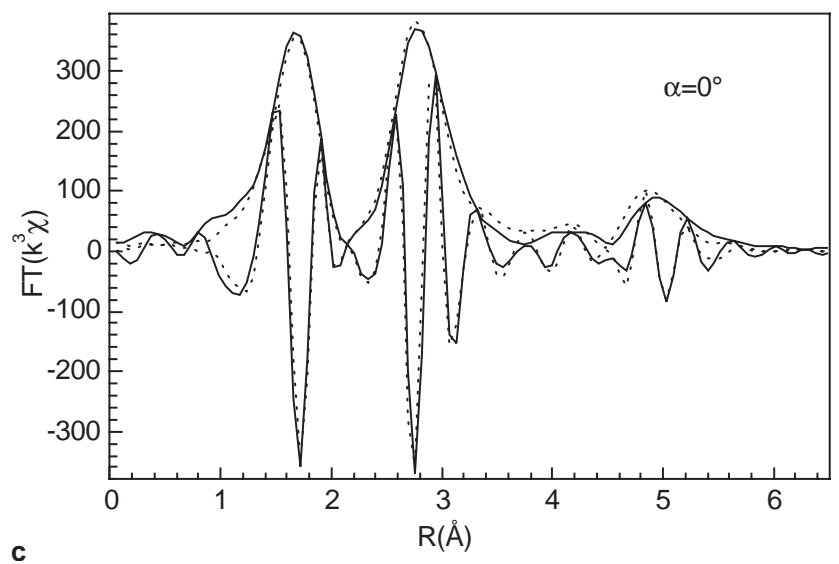

Manceau and Calas 1986; O'Day et al. 1994). The general correspondence between experimental and theoretical FTs indicates that the DVLS-derived structural model for nontronite is correct, and demonstrates the capacity of FEFF to simulate Fe K-edge P-EXAFS spectra of layer silicates.

\section{Higher shells analysis}

Up to now most of EXAFS studies on phyllosilicates were restricted to the analysis of the three nearest atomic shells below $R \approx 3.3 \AA$, and represented by the ligand and nearest

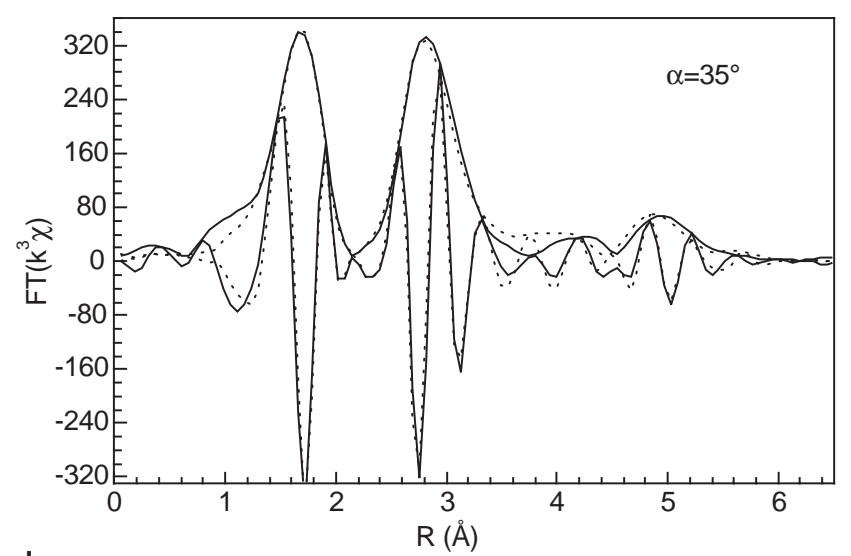

d

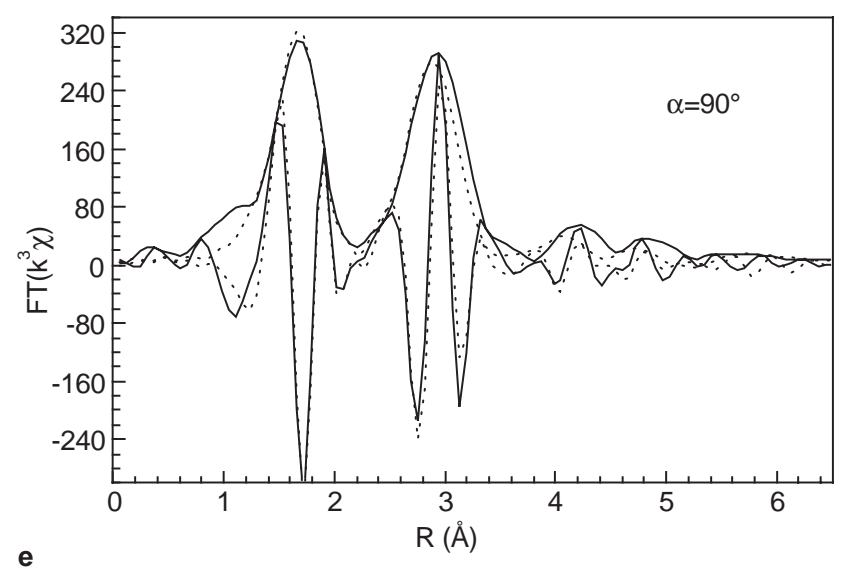

Fig. 14 a FEFF Fourier transforms for $\alpha=0,35.3$, and $90^{\circ}$. The $e n-$ velope curves are the magnitude of the transforms (RSFs) and the fast oscillatory curves are the imaginary parts of the transforms. b-e Comparison of experimental (solid line) and FEFF Fourier transforms (dotted line)

octahedral $\left(\mathrm{Fe}_{1}\right)$ and tetrahedral $\left(\mathrm{Si}_{1}\right)$ cations. Examination of longer distance EXAFS contributions is difficult for several reasons. First, the structural analysis of more distant shells requires high quality spectra otherwise structural peaks are affected in real space by nonstructural features coming from the Fourier transform of high frequency noise. Second, the 3.3-6 A region contains predominantly oxygen atoms. Their scattering contribution partly overlaps, and masks the signal from next-nearest tetrahedral and octahedral cations. This is typically a situation where polarized experiments are warranted as they may reinforce the signal coming from a specific direction permitting separation of the interfering atomic shell con- 

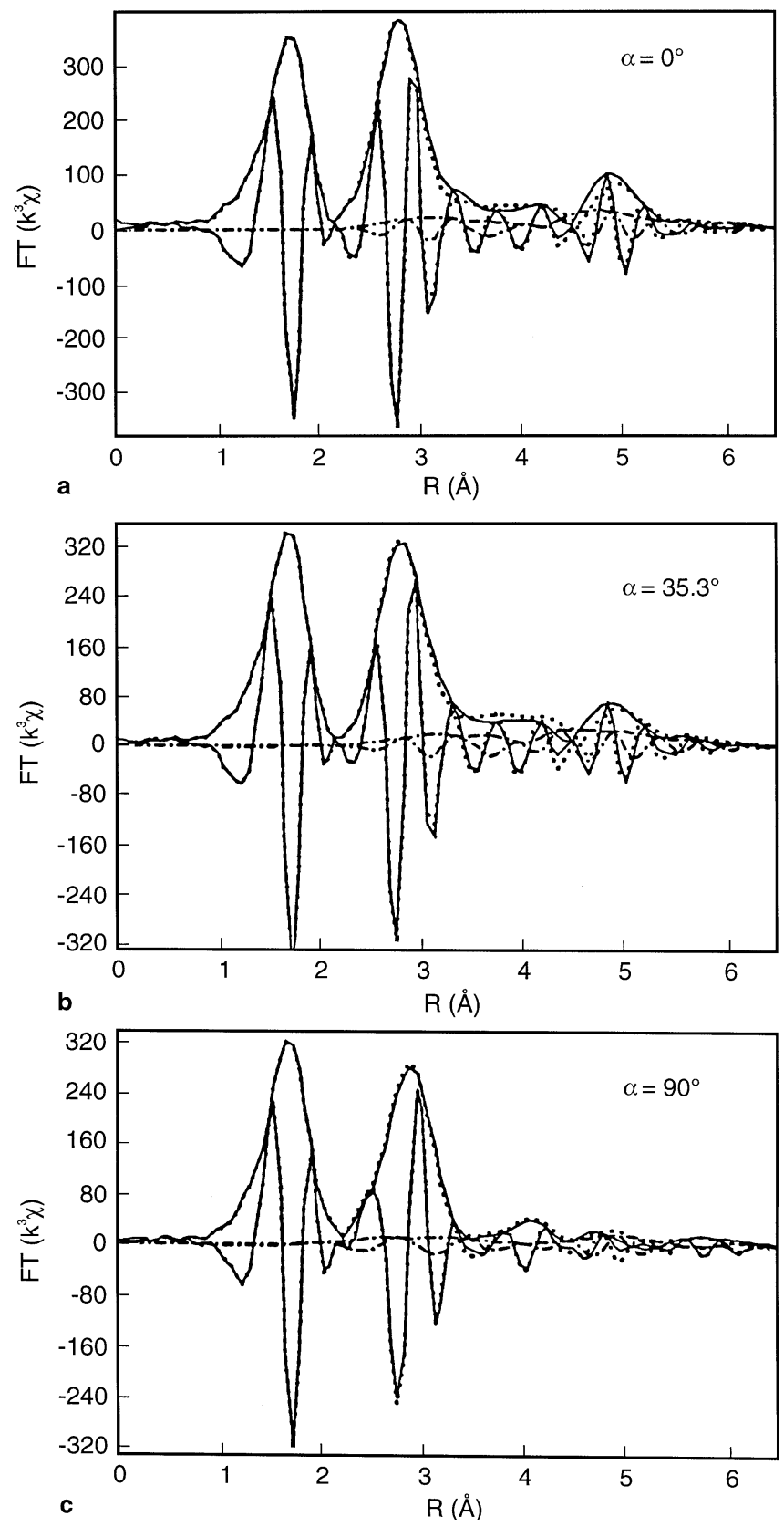

Fig. 15 Fourier transform deconvolution of the relative contributions of single-scattering $\left(\chi_{2}\right.$, dotted line $)$ and multiple-scattering $\left(\chi_{3-4}\right.$, half-dotted line) paths calculated by FEFF for $\alpha=0^{\circ}$ (a), $35.3^{\circ}(\mathrm{b})$, and $90^{\circ}(\mathrm{c})$. Full line $=\mathrm{SS}+\mathrm{MS}$

tributions. Third, this region is affected by multiple-scattering (MS) paths of the photoelectron. The FEFF allows us now to calculate with reasonable accuracy the contribution of single- and multiple-scattering paths in complex compounds. In the following sections, we reveal how the complement of three conditions, high quality spectra, angular measurements, and ab initio calculations, permits quantitative analysis of long-distance EXAFS contributions in highly oriented films of layer silicates for the first time.
Results of the full multiple-scattering path analysis obtained from FEFF calculations are shown in Fig. 15. To appreciate better the relative importance of SS and MS contributions to $\mathrm{FT}\left(k^{3} \chi(k)\right)$ functions, we have added all the computed MS signals and compared their Fourier transform to that of SS paths and to experimental spectra. In Fig. 15 MS have a fairly low intensity throughout the R range, and peaks $\mathrm{C}, \mathrm{D}$, and $\mathrm{E}$ result predominantly from SS paths. Examination of individual SS paths revealed that peak $\mathrm{C}$ corresponds to $(\mathrm{O} 1, \mathrm{O} 4)_{2}$ atoms at $R=3.74$ $3.82 \AA$, coordinated to neighboring edge-sharing $\mathrm{Fe}$ atoms (Table 2, Fig. 12). This shell makes a $\phi$ angle of $73^{\circ} \mathrm{ex}-$ plaining its magnification at $\alpha=0^{\circ}$. Peak E in Fig. 11 is predominantly due to the 6 next-nearest $\mathrm{Fe}$ atoms $\left(\mathrm{Fe}_{2}\right)$ at $5.28 \AA$ along [110], [1110], and [100] (Fig. 12). The location of Fe atoms in the octahedral sheet obviously accounts for the large anisotropy observed between the inplane and out-of-plane peak amplitude. However, one may note that this peak is not completely extinguished at $\alpha=90^{\circ}$, and a residual contribution is observed at $R+\Delta R=4.8-5.0 \AA$. This weak feature results from MS paths (Fig. 15c), and from the contribution of interlayer $\mathrm{Na}$ and of the $(\mathrm{Si}, \mathrm{Al})_{3}$ shell (Table 2). Lastly, the comparison of theoretical and experimental results showed that peak $\mathrm{D}$ corresponds to the $(\mathrm{Si}, \mathrm{Al})_{2}$ shell at $R=4.49 \AA$ (Table 2, Fig. 12). This peak has a weak angular dependence as $\phi=52^{\circ}$. Despite the fact that this angle is only $2.7^{\circ}$ lower than the magic angle, the dichroic effect is well detected. This observation is made possible owing to the high quality of spectral records, which allowed an accurate angular regression analysis. Additionally, this result confirms the high sensitivity of the method for measuring $\phi$ as it was shown previously for the flattening $\psi$ angle.

\section{Differentiation of dioctahedral and trioctahedral structures}

In trioctahedral phyllosilicates, all octahedral positions are occupied, whereas only $2 / 3$ of all available sites are in dioctahedral phyllosilicates (Fig. 1b). These two groups of minerals can be a priori differentiated by EXAFS by quantitative analysis of the nearest cation shell, as the octahedral cation $(\mathrm{Me})$ is surrounded by $6 \mathrm{Me}$ in the former, and $3 \mathrm{Me}$ in the latter. But when the octahedral sheet contains simultaneously $\mathrm{Fe}^{2+} / \mathrm{Fe}^{3+}$ and $\mathrm{Mg} / \mathrm{Al}$ cations, spectral fitting can lead to inaccurate structural interpretation (Manceau 1990). Indeed, because electronic waves backscattered by $\mathrm{Fe}$ and $\mathrm{Al} / \mathrm{Mg}$ are out of phase, and $\mathrm{Fe}$ atoms contain approximately twice as many electrons as $\mathrm{Al} / \mathrm{Mg}$, a dioctahedral local environment consisting of $3 \mathrm{Fe}^{3+}$, and a trioctahedral environment consisting of $4 \mathrm{Fe}^{2+}+2 \mathrm{Mg} / \mathrm{Al}$ result in approximately the same EXAFS signal. Thus, Manceau (1990) recommended that di- trioctahedral nature of layer silicates be accomplished by an alternative method in combination with EXAFS for studying the distribution of cations in octahedral sheets. It will be shown below that this question can be addressed 

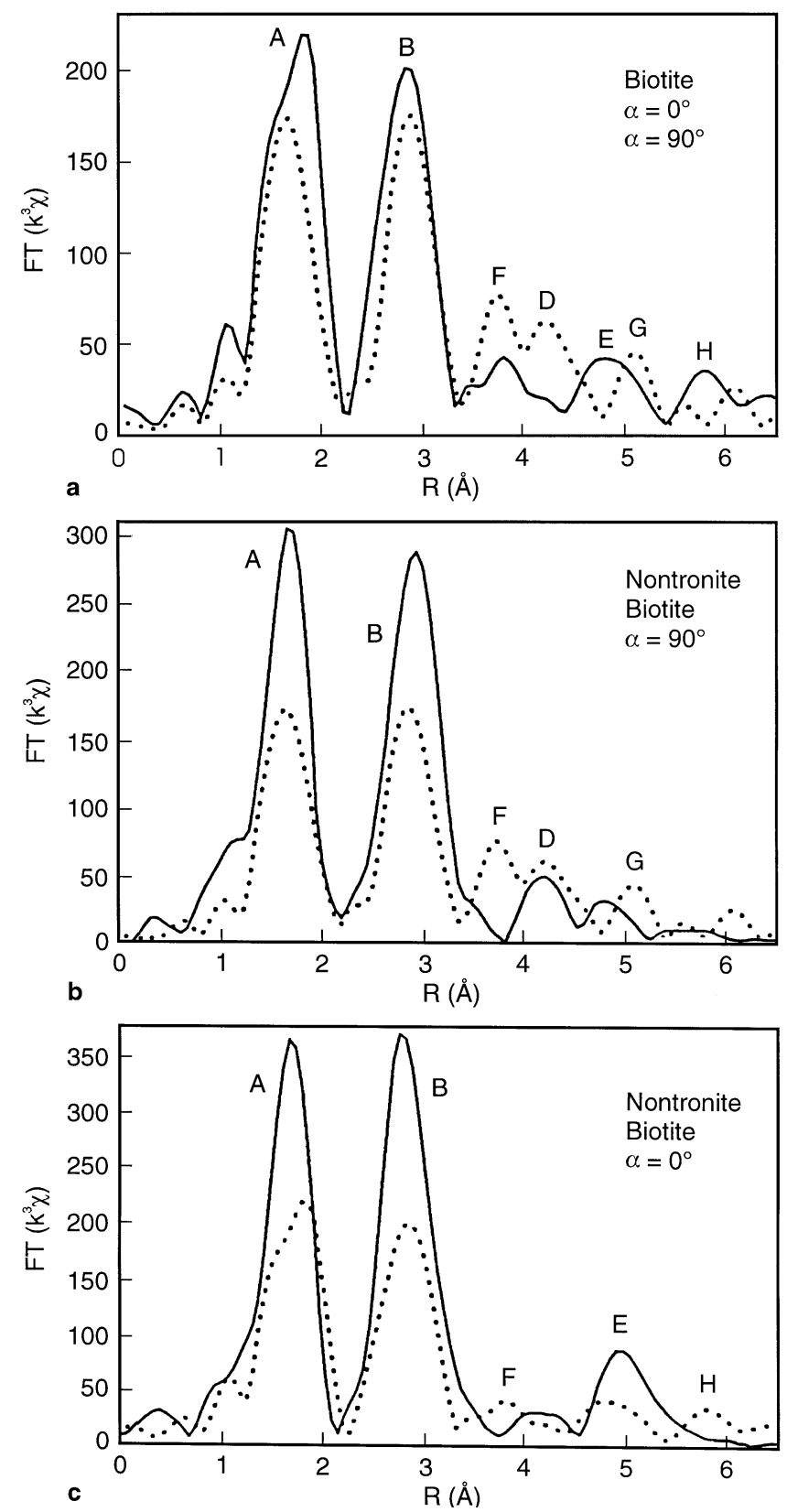

Fig. 16 a The $k^{3}$-weighted RSF for biotite at $\alpha=0^{\circ}$ (full line) and $90^{\circ}$ (dotted line). b-c Comparison of RSFs for Garfield nontronite (full line) and biotite (dotted line)

sensibly by examining atomic pair correlations in the 3.5$6.5 \AA$ distance range.

In Fig. 16 are contrasted the RSF for a trioctahedral biotite (\#B13 in Manceau et al. 1990) and for the Garfield nontronite. Comparison of the amplitude obviously shows lower A and B peaks in biotite compared to nontronite, despite the presence of six nearest Me cations in the former mineral. This effect partly results from a difference of static disorder between the two phyllosilicates. The composition of this biotite sample is $\left(\mathrm{Si}_{5.6} \mathrm{Al}_{2.4}\right)$ $\left(\mathrm{Fe}_{0.4}^{3+} \mathrm{Fe}_{2.5}^{2+} \mathrm{Al}_{1.0} \mathrm{Mg}_{1.1} \mathrm{Ti}_{0.3} \mathrm{Mn}_{0.2} \mathrm{Li}_{0.2}\right) \quad \mathrm{K}_{\sim 2} \mathrm{O}_{\sim 20}(\mathrm{OH}, \mathrm{F})_{\sim 4}$ (Sanz et al. 1984). The complex chemical composition of the octahedral sheet, as well as $\mathrm{Fe}^{3+}$ for $\mathrm{Fe}^{2+}$ substitutions, produces octahedral distortions. As a result, M1 and M2 sites are not regular and do not have similar dimensions as in other crystals. This distortion extends to nearest $\mathrm{Fe}-\mathrm{Me}$ distances which were found to vary from 2.97 to $3.13 \AA$ in the $1 M$ biotite from Ruiz Peak, New Mexico (Takeda and Ross 1975). In nontronite, the Fe-Fe1 distances are all equal to $3.05 \AA$, and the difference in coherency of the metal-metal distances between nontronite and biotite partly accounts for the different amplitudes observed for peak B. As explained in the previous section, the mixing of $\mathrm{Mg}$ and $\mathrm{Fe}$ atoms in the nearest cation shell also contributes to drop the amplitude of peak B in biotite owing to the phase contrast between $\mathrm{Fe}$ and $\mathrm{Mg}$.

Inspection of Fig. 16a shows that the RSF for biotite exhibits three additional peaks, labeled F, G, and $\mathrm{H}$, not observed for nontronite. These three peaks exhibit a very strong dichroic dependence: peaks $\mathrm{G}$ and $\mathrm{H}$ are observed at only one orientation, and peak $\mathrm{F}$ is strongly diminished at $\alpha=0^{\circ}$. Peak $\mathrm{G}$ was identified as arising from the $(\mathrm{Si}, \mathrm{Al})_{3}$ shell at 5.4-5.5 $\AA$, and from interlayer $\mathrm{K}$ at $5.30 \AA$ $\left(\phi=19^{\circ}\right.$, Takeda and Ross 1975). This peak is shifted to lower distance (peak E, Fig. 16b) in Na-nontronite as a result of the shorter $\mathrm{Fe}-\mathrm{Na}$ distance $(5.12 \AA)$. It has also a lower intensity for at least three reasons: (1) $\mathrm{Me}-(\mathrm{Si}, \mathrm{Al})_{3}$ distances are more incoherent in dioctahedral structures; (2) $\mathrm{Na}$ is a weaker scatterer; (3) $\mathrm{Fe}-\mathrm{Na}$ and $\mathrm{Fe}-(\mathrm{Si}, \mathrm{Al})_{3}$ waves are nearly out of phase $(\Delta R \sim 0.3 \AA)$. Unlike peak $\mathrm{G}$, peaks $\mathrm{F}$ and $\mathrm{H}$ originate from atoms located within the layer framework, and are much more sensitive to the di-trioctahedral character of the octahedral sheet.

Peak F corresponds to the contributions of nearest $\mathrm{O} 2$ $\left(\mathrm{O} 2_{1}\right)$ and next-nearest $\mathrm{O} 3\left(\mathrm{O}_{2}\right)$ of the tetrahedral basal plane (Fig. 12a). As discussed in the DVLS section, basal oxygens are at the same $z$ position in trioctahedral structures (Fig. 12c), and thus form an identical $\phi$ angle of $37^{\circ}$ in biotite (Takeda and Ross 1975). In addition, $\mathrm{Fe}-\mathrm{O} 2_{1}$ and $\mathrm{Fe}-\mathrm{O} 3_{2}$ distances are coherent $(\approx 4.15-4.20 \AA)$, and these two factors enhance the contribution of basal oxygens in the out-of-plane orientation. In dioctahedral structures, $\mathrm{O} 2{ }_{1}$ and $\mathrm{O} 3_{2}$ atoms undergo a vertical displacement to relieve the misfit between the octahedral sheet and the attached tetrahedral sheet. This corrugation of the oxygen basal plane (Fig. 12c) modifies the $\phi$ angle, and splits the $\mathrm{Fe}-\mathrm{O}$ distances causing the disappearance of peak $\mathrm{F}$ in the experimental RSFs of nontronite (Fig. 16b). This analysis infers that vacant octahedral sites can indirectly be detected by analyzing the out-of-plane structure of phyllosilicates. In our opinion, dioctahedral and trioctahedral layer silicates can more reliably be differentiated on the basis of this intrinsic structural criterion rather than by a curve fitting determination of the number of nearest layer $\mathrm{Me}-\mathrm{Me}$ pairs.

In the biotite RSF (Fig. 16), peak $\mathrm{H}$ is located at twice the distance of peak $\mathrm{B}(R+\Delta R=5.8 \AA)$, and thus corresponds to the third $\mathrm{Fe}$ shell $\left(\mathrm{Fe}_{3}\right)$ at $R \approx 6.2 \AA$. It is interesting to note that the more distant $\mathrm{Fe}_{3}$ shell has a similar amplitude as that of the $\mathrm{Fe}_{2}$ shell at $R=5.3 \AA$ (peak E). Since the amplitude of the EXAFS signal decreases as 


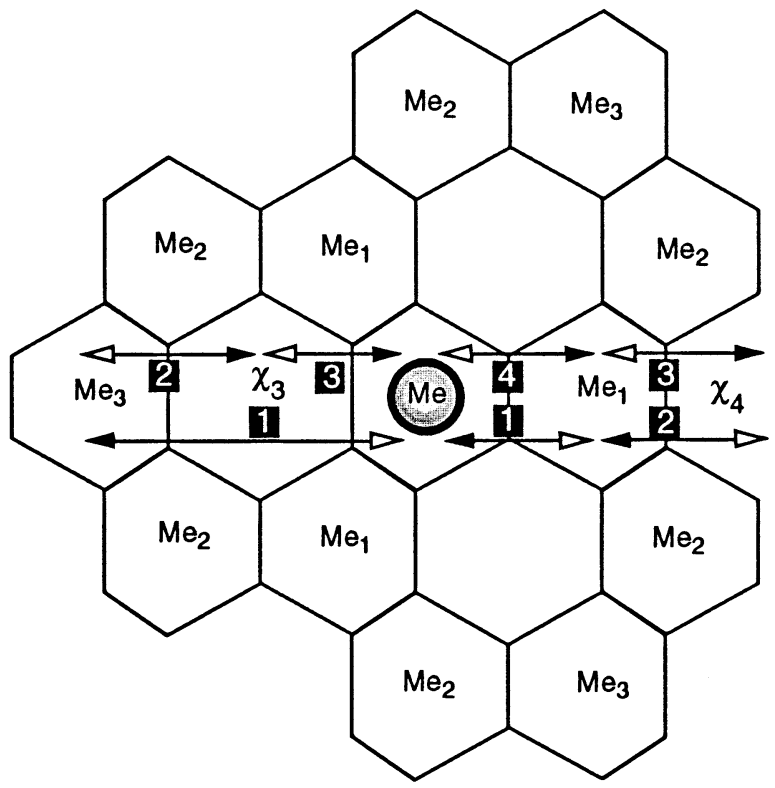

Fig. 17 Distribution of cations in di- and trioctahedral sheets, and representation of most intense $\chi_{3}$ and $\chi_{4}$ paths. Dioctahedral sheets have no $\mathrm{Me}-\mathrm{Me}_{1}-\mathrm{Me}_{3}$ sequences and, consequently, the intensity of $\chi_{3}$ and $\chi_{4}$ MS paths is very low

$1 / \mathrm{R}^{2}$, the $\mathrm{Fe}_{3}$ shell contribution would be expected to be diminished by $25 \%$. In addition, interatomic distance calculations for biotite (Takeda and Ross 1975) show that $\mathrm{Fe}-\mathrm{Fe}_{3}$ distances are much more incoherent $(6.10 \AA<R<6.26 \AA)$ than $\mathrm{Fe}-\mathrm{Fe}_{2}$ distances $(R=5.33 \AA)$. Thus, the greater disorder of the third $\mathrm{Fe}$ shell also lessens the intensity of $\mathrm{Fe}_{3}$ relative to $\mathrm{Fe}_{2}$. In fact, peak $\mathrm{H}$ has been shown to be amplified by a shadowing (focusing) MS effect between collinear octahedral cations (O'Day et al. 1994). The MS effect between outgoing and backscattered photoelectron waves, when pairs are aligned, increases the peak amplitude above that expected for a single outgoing and backscattering event.

$A b$ initio calculations showed that the two most intense scattering pathways arise from $\mathrm{Me} \rightarrow \mathrm{Me}_{1} \rightarrow \mathrm{Me}_{3} \rightarrow \mathrm{Me}$ or inverse $\mathrm{Me} \rightarrow \mathrm{Me}_{3} \rightarrow \mathrm{Me}_{1} \rightarrow \mathrm{Me}$ third order $\left(\chi_{3}\right)$ paths, and from the $\mathrm{Me} \rightarrow \mathrm{Me}_{1} \rightarrow \mathrm{Me}_{3} \rightarrow \mathrm{Me}_{1} \rightarrow \mathrm{Me}$ or inverse fourth order $\left(\chi_{4}\right)$ path (Fig. 17). Atom $\mathrm{Me}$ is the central atom, $\mathrm{Me}_{1}$ belongs to the nearest Me shell at 3.0-3.1 $\AA$, and $\mathrm{Me}_{3}$ to the 3 rd shell at $6.0-6.2 \AA$. The $\mathrm{Me}_{1} \leftrightarrow \mathrm{Me} \leftrightarrow \mathrm{Me}_{1}$ $\chi_{3}$ and $\chi_{4}$-paths, in which the central Me also actuates as a scatterer in the focusing geometry, are comparatively less intense. Figure 17 shows that none of these paths exists in dioctahedral structures as a result of the vacancy of one of the three octahedral sites involved in the focusing effect. The three $\mathrm{Me}_{3}$ atoms at twice the $\mathrm{Me}-\mathrm{Me}_{1}$ distance are separated from the central Me by a vacancy site, and their contribution is expected to be weak. This is precisely what is observed in Fig. 14c where the intensity of peak $\mathrm{H}$ for Garfield nontronite is $\approx 0$. As for the tilt of the basal $\mathrm{O} 2-\mathrm{O} 3$ shell, this second criterion for differentiating di-
Trioctahedral structure

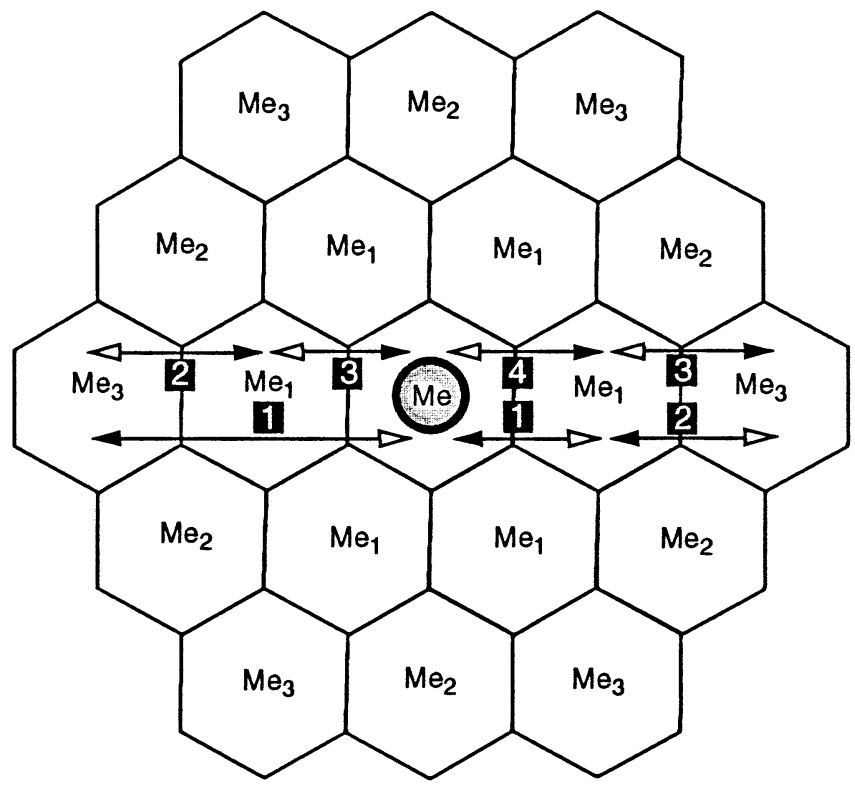

octahedral from trioctahedral structures can be applied to powder EXAFS spectra. However, the comparison of $\operatorname{FT}\left(\chi\left(\alpha=0^{\circ}\right)\right.$ and $\operatorname{FT}\left(\chi\left(\alpha=35^{\circ}\right)\right.$ in Fig. 11 clearly demonstrates that the sensitivity of this method is much improved in polarized experiments. Increasing this sensitivity may be important for the study of clays with an intermediate di- trioctahedral structure, i.e., for detecting the presence of small trioctahedral clusters within a dioctahedral framework.

Acknowledgments We thank V.A. Drits for insightful discussions of individual aspects of this paper. LURE laboratory is acknowledged for providing access to the synchrotron beam, and the French government for providing for living expenses (W.P.G.) through a bourse Chateaubriand.

\section{References}

Bailey SW (1984) Micas. (Reviews in mineralogy, Vol 13) Mineral Soc Am, Washington DC

Bailey SW (1991) Hydrous phyllosilicates. (Reviews in mineralogy, Vol 19) Mineral Soc Am, Washington DC

Bauer ED, Bridges F, Booth CH, Boyce JB, Claeson T, Brorsson G, Suzuki Y (1996) Local structure study about $\mathrm{Co}$ in $\mathrm{YBa}_{2}\left(\mathrm{Cu}_{1-x}\right.$ $\left.\mathrm{Co}_{x}\right)_{3} \mathrm{O}_{7-\delta}$ thin films using polarized XAFS. Phys Rev B54:13352-13360

Benfatto M, Natoli CR, Brouder C, Pettifer RF, Ruiz Lopez MF (1989) Polarized curved-wave extended X-ray-absorption fine structure: theory and application. Phys Rev B39:1936-1939

Besson G, Bookin AS, Dainak LG, Rautureau M, Tsipursky SI, Tchoubar C, Drits VA (1983) Use of diffraction and Mössbauer methods for the structural and crystallochemical characterization of nontronites. J Appl Crystallogr 16:374-183

Bish DL, Von Dreele RB (1989) Rietveld refinement of non-hydrogen atomic positions in kaolinite. Clays Clay Miner 37:289-296

Bonnin D, Calas G, Suquet H, Pezerat H (1985) Sites occupancy of $\mathrm{Fe}^{3+}$ in Garfield nontronite: a spectroscopic study. Phys Chem Miner 12:55-64

Bonnin D, Bouat J, Kaiser P, Frétigny C, Béguin F (1986) Bond angle determination by angular EXAFS study and Debye-Waller 
anisotropy in 2D graphite intercalation compounds. In: Lagarde P, Raoux D, Petiau J (eds) EXAFS and near edge structure IV. J Phys Paris, pp 865-868

Borchardt G (1995) Smectites. In: Dixon JB, Weed SB (eds) Minerals in soil environments. Soil Sci Soc Am Book Ser, Madison Wisconsin, pp 675-728

Brese NE, O'Keefe M (1991) Bond-valence parameters for solids. Acta Crystallogr B47:192-197

Brouder C (1990) Angular dependence of X-ray absorption spectra. J Phys Cond Matter 2:701-738

Brown ID (1981) The bond-valence method: an empirical approach to chemical structure and bonding. In: O'Keefe M, Navrotsky A (eds) Structures and bonding in crystals. Academic Press, New York, pp 1-30

Brown ID (1992) Chemical and steric constraints in inorganic solids. Acta Crystallogr B48:553-572

Brown ID, Altermatt D (1985) Bond-valence parameters obtained from a systematic analysis of the inorganic crystal structure database. Acta Crystallogr B41:244-247

Brown ID, Shannon RD (1973) Empirical bond-strength-bondlength curves for oxides. Acta Crystallogr A29:266-282

Bunge HJ (1981) Textures in materials science. Butterworths, London

Chaboy J, Garcia J (1996) Theoretical analysis of the multiple-scattering contributions to the extended $\mathrm{X}$-ray absorption fine-structure spectra at the barium $\mathrm{L}_{1}$ and $\mathrm{L}_{3}$ edges in $\mathrm{BaF}_{2}$. J Phys Cond Matter 8:5659-5671

Chateigner D, Wenk HR, Pernet M (1997) Orientation analysis of bulk YBCO from incomplete neutron diffraction data. J Appl Crystallogr 30:43-48

Eggleton RA (1977) Nontronite: chemistry and X-ray diffraction. Clay Miner 12:181-194

Guggenheim S, Eggleton RA (1994) A comparison of the structures and geometric stabilities of stilpnomelane and parsettensite - a distance least-squares (DLS) study. Am Mineral 79:438-442

Güven N (1991) Smectites. In: Bailey SW (ed) Hydrous phyllosilicates. (Reviews in mineralogy, Vol 19) Mineral Soc Am, Washington DC, pp 497-560

Hazemann JL, Manceau A, Sainctavit P, Malgrange C (1992) Structure of the $\alpha \mathrm{Fe}_{\mathrm{x}} \mathrm{Al}_{1-\mathrm{x}} \mathrm{OOH}$ solid solution. I. Evidence by polarized EXAFS for an epitaxial growth of hematite-like clusters in diaspore. Phys Chem Miner 19:25-38

Heald SM, Stern EA (1977) Anisotropic X-ray absorption in layered compounds. Phys Rev B16:5549-5559

Kroll H, Maurer H, Stöckelmann D, Beckers W, Fulst J, Krüsemann R, Stutenbäumer T, Zingel A (1992) Simulation of crystal structures by a combined distance-least-squares/valence-rule method. Z Kristallogr 199:49-66

Lederer T, Arvanitis D, Tischer M, Comelli G, Troger L, Baberschke K (1993) Structural determination of $\mathrm{c}(2 \times 2) \mathrm{N} / \mathrm{Cu}(100)$ : a multiple-scattering surface-EXAFS study. Phys Rev B48:11277

Lee JH, Guggenheim (1981) Single crystal X-ray refinement of pyrophyllite-1Tc. Am Mineral 66:350-357

Lengeler B, Eisenberger P (1980) Extended X-ray absorption fine structure analysis of interatomic distances, coordination numbers, and mean relative displacements in disordered alloys. Phys Rev B21:4507-4528

Lu K, Stern EA (1983) Size effect of powdered sample on EXAFS amplitude. Nucl Instrum Methods 212:475-478

Manceau A (1990) Distribution of cations among the octahedra of phyllosilicates: insight from EXAFS. Can Mineral 28:321-328

Manceau A, Calas G (1986) Ni-bearing clay minerals. 2. X-ray absorption study of $\mathrm{Ni}-\mathrm{Mg}$ distribution. Clay Miner 21:341360
Manceau A, Combes JM (1988) Structure of Mn and Fe oxides and oxyhydroxides: a topological approach by EXAFS. Phys Chem Miner 15:283-295

Manceau A, Gates W (1997) Surface structural model for ferrihydrite. Clays Clay Miner 43:448-460

Manceau A, Bonnin D, Kaiser P, Frétigny C (1988) Polarized EXAFS of biotite and chlorite. Phys Chem Miner 16:180-185

Manceau A, Bonnin D, Stone WEE, Sanz J (1990) Distribution of Fe in the octahedral sheet of trioctahedral micas by polarized EXAFS. comparison with NMR results. Phys Chem Miner 17:363370

Matthies SM, Vinel GW, Helming K (1987) Standard distributions in texture analysis. Akad Verlag, Berlin

Meier WM, Villiger H (1969) Die Methode der Abstandsverfeinerung zur Bestimmung der Atomkoordinaten idealisierter Gerüstrukturen. Z Kristallogr 129:411-423

Méring J, Oberlin A (1967) Electron-optical study of smectites. Clays Clay Miner 15:3-25

Murad E (1987) Mössbauer spectra of nontronites: structural implications and characterization of associated iron oxides. Zeits Pflanzenernaehr Bodenk 279-285

O'Day PA, Rehr JJ, Zabinsky SI, Brown GE Jr (1994) Extended Xray absorption fine structure (EXAFS) analysis of disorder and multiple-scattering in complex crystalline solids. J Am Chem Soc 116:2938-2949

Pauling L (1929) The principles determining the structure of complex ionic crystals. J Am Chem Soc 51:1010-1026

Rehr JJ, Mustre de Leon J, Zabinsky SI, Albers RC (1991) Theoretical X-ray absorption fine structure standards. J Am Chem Soc 113:5135-5145

Rehr JJ, Zabinsky SI, Albers RC (1992) High-order multiple-scattering calculations of X-ray-absorption fine structure. Phys Rev Lett 69:3397

Sanz J, de la Calle C, W.E.E. S (1984) NMR applied to minerals. V. The localization of vacancies of the octahedral sheet of aluminous biotites. Phys Chem Miner 11:235-240

Schulz LG (1949) J Appl Phys 20:1030

Stern EA, Heald SM (1983) Basic principles and applications of EXAFS. In: Koch E (ed) Handbook of synchrotron radiation. North-Holland Amsterdam, New York, pp 955-1014

Stern EA, Kim K (1981) Thickness effect on the extended X-ray absorption fine structure amplitude. Phys Rev B23:3781-3787

Stöhr J (1992) NEXAFS spectroscopy. Springer-Verlag, Berlin Heidelberg New York Tokyo

Stucki JW (1988) Structural iron in smectites. In: Stucki JW, Goodman BA, Schwertmann U (eds) Iron in soils and clay minerals. (NATO ASI Series, 217) Riedel Publishing Co, Dordrecht, pp 625-676

Takeda H, Ross M (1975) Mica polytypism: dissimilarities in the crystal structures of coexisting $1 \mathrm{M}$ and $2 \mathrm{M} 1$ biotite. Am Mineral 60: $1030-1040$

Tsipursky SI, Drits VA (1984) The distribution of cations in the 2:1 layers of dioctahedral smectites studied by oblique-texture electron diffraction. Clay Miner 19:177-193

Wenk HR (1985) Measurement of pole figures. In: Wenk HR (ed) Preferred orientation in deformed metals and rocks. Academic Press Inc, Orlando, pp 11-47

Wenk HR, Chateigner D, Pernet M, Bingert J, Hellstrom E, Ouladdiaf B (1996) Texture analysis of Bi-2212 and 2223 tapes and wires by neutron diffraction. Physica C 272:1-12

Wenk HR, Matthies S, Donovan J, Chateigner D (1997) A Windows-based program system for quantitative texture analysis. J Appl Crystallogr (in press) 